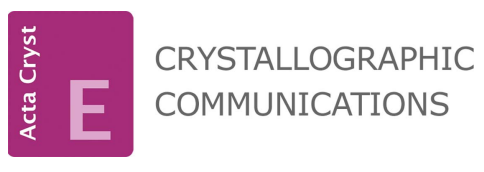

ISSN 2056-9890

Received 13 April 2020

Accepted 26 April 2020

Edited by A. M. Chippindale, University of Reading, England

Keywords: crystal structure; $\mathrm{Lu}_{4} \mathrm{Al}_{2} \mathrm{O}_{9}$; lutetium aluminate monoclinic (LUAM); rare-earth aluminate; $\mathrm{Lu}_{2} \mathrm{O}_{3}-\mathrm{Al}_{2} \mathrm{O}_{3}$ system; single-crystal $\mathrm{X}$-ray diffraction.

CCDC reference: 1999289

Supporting information: this article has supporting information at journals.iucr.org/e
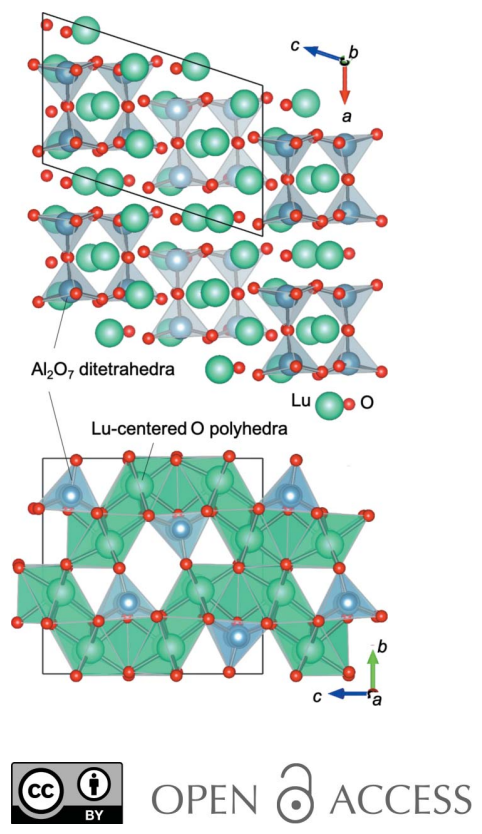

\section{Crystal structure of lutetium aluminate (LUAM), $\mathrm{Lu}_{4} \mathrm{Al}_{2} \mathrm{O}_{9}$}

\author{
Rayko Simura* and Hisanori Yamane
}

Institute of Multidisciplinary Research for Advanced Materials, Tohoku, University, 2-1-1 Katahira, Aoba-ku, Sendai, 9808577, Japan.*Correspondence e-mail: ray@tohoku.ac.jp

The crystal structure of the title compound containing lutetium, the last element in the lanthanide series, was determined using a single crystal prepared by heating a pressed pellet of a 2:1 molar ratio mixture of $\mathrm{Lu}_{2} \mathrm{O}_{3}$ and $\mathrm{Al}_{2} \mathrm{O}_{3}$ powders in an $\mathrm{Ar}$ atmosphere at $2173 \mathrm{~K}$ for $4 \mathrm{~h} . \mathrm{Lu}_{4} \mathrm{Al}_{2} \mathrm{O}_{9}$ is isostructural with $\mathrm{Eu}_{4} \mathrm{Al}_{2} \mathrm{O}_{9}$ and composed of $\mathrm{Al}_{2} \mathrm{O}_{7}$ ditetrahedra and Lu-centered six- and sevenfold oxygen polyhedra. The unit-cell volume, 787.3 (3) $\AA^{3}$, is the smallest among the volumes of the rare-earth $(R E)$ aluminates, $R E_{4} \mathrm{Al}_{2} \mathrm{O}_{9}$. The crystal studied was refined as a two-component pseudo-merohedric twin.

\section{Chemical context}

In the $\mathrm{Al}_{2} \mathrm{O}_{3}-\mathrm{Lu}_{2} \mathrm{O}_{3}$ system, where $\mathrm{Lu}$ has the largest atomic number among the rare-earth elements $(R E)$, the following three phases have been reported: $\mathrm{Lu}_{3} \mathrm{Al}_{5} \mathrm{O}_{12}, \mathrm{LuAlO}_{3}$, and $\mathrm{Lu}_{4} \mathrm{Al}_{2} \mathrm{O}_{9}$. These phases have been actively investigated as host materials, not only for phosphors (Ding et al., 2011; Xiang et al., 2016; Wang et al., 2018), but also for scintillators, owing to their large radiation absorption cross sections arising from the presence of $\mathrm{Lu}$. Various scintillation properties of $\mathrm{Ce}$ - and Pr-doped $\mathrm{Lu}_{3} \mathrm{Al}_{5} \mathrm{O}_{12}$ and $\mathrm{LuAlO}_{3}$ crystals have been characterized (Wojtowicz, 1999; Nikl, 2000; Wojtowicz et al., 2006; Nikl et al., 2013), and the luminescence properties of Ce- and Pr-doped $\mathrm{Lu}_{4} \mathrm{Al}_{2} \mathrm{O}_{9}$ evaluated (Lempicki et al., 1996; Zhang et al., 1997, Zhang et al., 1998; Drozdowski et al., 2005). The crystal structures of the lutetium aluminates $\mathrm{Lu}_{3} \mathrm{Al}_{5} \mathrm{O}_{12}$ (Euler \& Bruce, 1965) and $\mathrm{LuAlO}_{3}$ (Dernier \& Maines, 1971; Shishido et al., 1995) have been determined as garnet-type (LUAG) and perovskite-type (LUAP), respectively. However, to date, there have been no reports of the lattice constants of $\mathrm{Lu}_{4} \mathrm{Al}_{2} \mathrm{O}_{9}$, although Shirvinskaya \& Popova (1977) treated it as isotypic with $\mathrm{Y}_{4} \mathrm{Al}_{2} \mathrm{O}_{9}$ and have reported the $d$-spacings and relative peak intensities in the powder $\mathrm{X}$-ray diffraction pattern (PDF\#00-033-0844).

Many $R E \mathrm{Al}_{2} \mathrm{O}_{9}$ compounds have been investigated in detail. After Warshaw \& Roy (1959) first reported the existence of $\mathrm{Y}_{4} \mathrm{Al}_{2} \mathrm{O}_{9}$, Reed \& Chase (1962) determined the space group of this material as $P 2{ }_{1} / c$ using X-ray Weissenberg and precession photography. Christensen \& Hazell (1991) later determined the crystal structure of $\mathrm{Y}_{4} \mathrm{Al}_{2} \mathrm{O}_{9}$ using powder synchrotron X-ray and neutron diffraction. Brandle \& Steinfink (1969) also prepared crystals of $R E \mathrm{Al}_{2} \mathrm{O}_{9}(R E=\mathrm{Sm}, \mathrm{Gd}$, $\mathrm{Eu}, \mathrm{Dy}, \mathrm{Ho})$ and determined the crystal structure of $\mathrm{Eu}_{4} \mathrm{Al}_{2} \mathrm{O}_{9}$ using X-ray diffraction. 
The lattice parameters of $R E_{4} \mathrm{Al}_{2} \mathrm{O}_{9}$ have previously been reported for $R E=\mathrm{Y}$ (Lehmann et al., 1987; Reed \& Chase, 1962; Christensen \& Hazell, 1991; Yamane et al., 1995b; Talik et al., 2016), La (Dohrup et al., 1996), Pr (Dohrup et al., 1996), Nd (Dohrup et al., 1996), Sm (Brandle \& Steinfink, 1969; Mizuno et al., 1977a; Yamane et al., 1995a), Eu (Brandle \& Steinfink, 1969; Mizuno et al., 1977b; Yamane et al., 1995a), Gd (Brandle \& Steinfink, 1969; Mizuno et al., 1977b; Yamane et al., 1995a; Dohrup et al., 1996; Martín-Sedeño et al., 2006), Tb (Jero \& Kriven, 1988; Yamane et al., 1995a; Dohrup et al., 1996; Li et al., 2009), Dy (Brandle \& Steinfink, 1969; Mizuno et al., 1978; Yamane et al., 1995a), Ho (Brandle \& Steinfink, 1969; Mizuno, 1979; Yamane et al., 1995a), Er (Mizuno, 1979; Yamane et al., 1995a), Tm (Yamane et al., 1995a), and Yb (Mizuno \& Noguchi, 1980; Yamane et al., 1995a).

Wu \& Pelton (1992) investigated the phase diagram of the $\mathrm{Lu}_{2} \mathrm{O}_{3}-\mathrm{Al}_{2} \mathrm{O}_{3}$ system and showed that $\mathrm{Lu}_{4} \mathrm{Al}_{2} \mathrm{O}_{9}$ melted congruently at $2313 \mathrm{~K}$ under an inert atmosphere. Petrosyan $e t$ al. (2006) studied the same system under a reducing atmosphere and reported that $\mathrm{Lu}_{4} \mathrm{Al}_{2} \mathrm{O}_{9}$ could be formed by reaction of $\mathrm{Lu}_{2} \mathrm{O}_{3}$ and $\mathrm{Lu}_{3} \mathrm{Al}_{5} \mathrm{O}_{12}$ at $1923 \mathrm{~K}$, but decomposed into $\mathrm{Lu}_{2} \mathrm{O}_{3}$ and a melt at $2273 \mathrm{~K}$. Subsequently, Petrosyan et al. (2013) observed incongruent melting of $\mathrm{Lu}_{4} \mathrm{Al}_{2} \mathrm{O}_{9}$ at $2123 \mathrm{~K}$ under an $\mathrm{Ar} / 2 \% \mathrm{H}_{2}$ atmosphere using differential thermal analysis (DTA). Klimm (2010) employed DTA to investigate $\mathrm{LuAlO}_{3}$ melting behavior in a $5 \mathrm{~N}$ pure Ar flow and concluded that the congruent and incongruent melting of $\mathrm{LuAlO}_{3}$ depended on the atmospheric conditions. The author also concluded that the phase diagram at around $\mathrm{Lu}: \mathrm{Al}=1: 1$ under an inert atmosphere, previously reported by $\mathrm{Wu} \&$ Pelton (1992), is correct. Yamane et al. (1995a) reported that only a very small amount of $\mathrm{Lu}_{4} \mathrm{Al}_{2} \mathrm{O}_{9}$ can be obtained by reactions in air at $1673-2073 \mathrm{~K}$, even though $R E_{4} \mathrm{Al}_{2} \mathrm{O}_{9}(R E=$ $\mathrm{Y}, \mathrm{Sm}-\mathrm{Yb}$ ) can be synthesized under the same conditions.

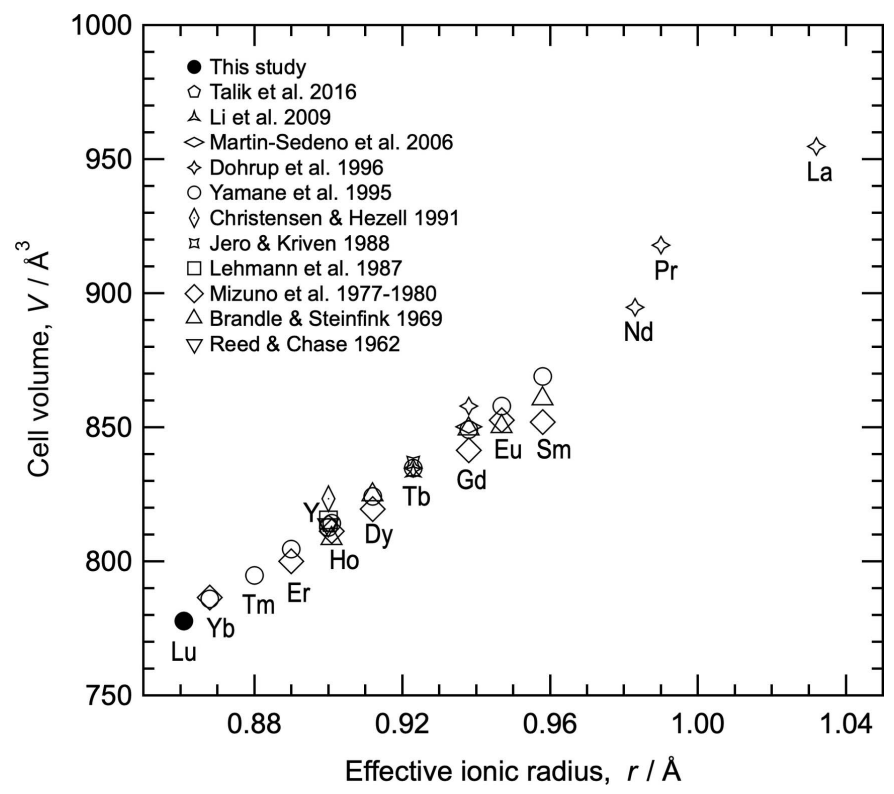

Figure 1

Unit-cell volume of $R E_{4} \mathrm{Al}_{2} \mathrm{O}_{9}$ versus effective ionic radius for the trivalent rare-earth anion $\left(R E^{3+}\right)$ with sixfold coordination.

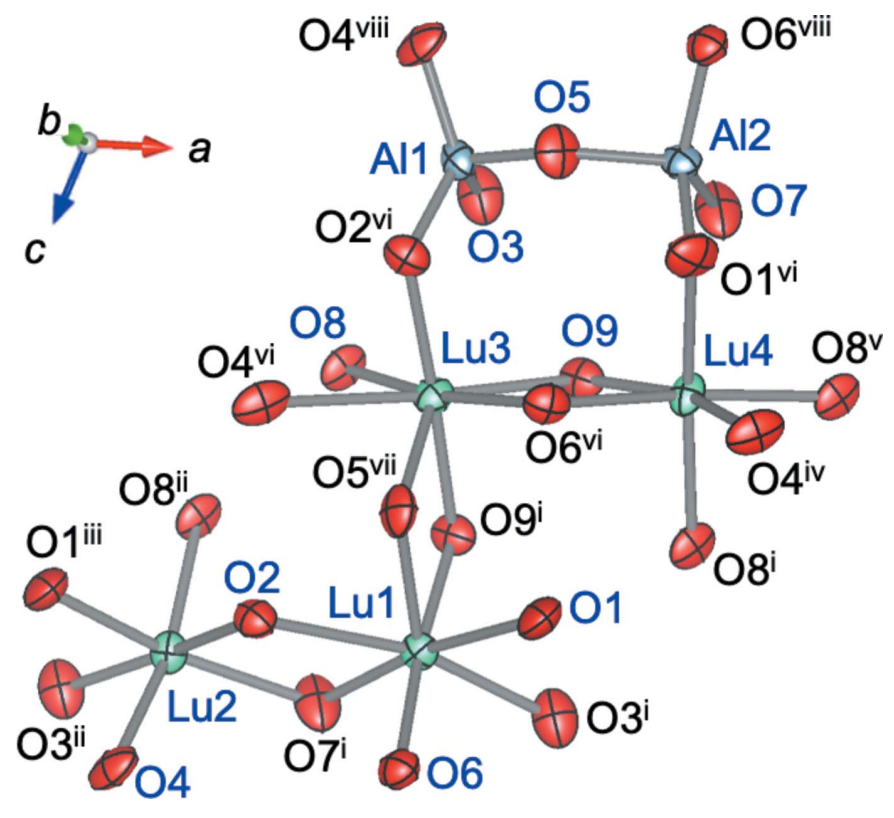

Figure 2

The atomic arrangement of $\mathrm{Lu}_{4} \mathrm{Al}_{2} \mathrm{O}_{9}$ depicted with displacement ellipsoids at the $99 \%$ probability level. [Symmetry codes: (i) $1-x,-y$, $1-z$; (ii) $-x,-y, 1-z$; (iii) $x-1, y, z$; (iv) $1+x,-y+\frac{1}{2}, z-\frac{1}{2}$; (v) $x+1, y$, $z$; (vi) $x,-y+\frac{1}{2}, z-\frac{1}{2}$; (vii) $x,-y+\frac{1}{2}, z+\frac{1}{2}$; (viii) $x, y, z-1$.]

Following these reports, the present authors also attempted to synthesize $\mathrm{Lu}_{4} \mathrm{Al}_{2} \mathrm{O}_{9}$ by heating a 2:1 molar ratio powder mixture of $\mathrm{Lu}_{2} \mathrm{O}_{3}$ and $\mathrm{Al}_{2} \mathrm{O}_{3}$ at $2073 \mathrm{~K}$ for $2 \mathrm{~h}$ in air, but the sample obtained was a mixture of $\mathrm{LuAlO}_{3}$ and $\mathrm{Lu}_{2} \mathrm{O}_{3}$ (see Fig. S1 $a$ in the supporting information). The method used to prepare the single crystals of $\mathrm{Lu}_{4} \mathrm{Al}_{2} \mathrm{O}_{9}$ used for the present diffraction study is described below.

\section{Structural commentary}

$\mathrm{X}$-ray diffraction spots from the $\mathrm{Lu}_{4} \mathrm{Al}_{2} \mathrm{O}_{9}$ single crystal were indexed on the basis of a monoclinic unit cell with lattice

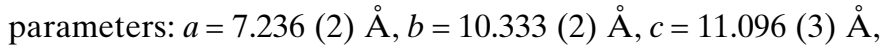
and $\beta=108.38(2)^{\circ}$. As shown in Fig. 1, the unit-cell volume of $\mathrm{Lu}_{4} \mathrm{Al}_{2} \mathrm{O}_{9}$ calculated from these parameters lies on the extrapolated line of $R E_{4} \mathrm{Al}_{2} \mathrm{O}_{9}$ volumes plotted against the effective ionic radii for sixfold coordination of the trivalent rare-earth anions $\left(R E^{3+}\right)$ (Shannon, 1976). In other words, $\mathrm{Lu}_{4} \mathrm{Al}_{2} \mathrm{O}_{9}$ containing $\mathrm{Lu}$, which has the smallest effective ionic radius of the $R E$ atoms, has the smallest unit-cell volume in the $R E_{4} \mathrm{Al}_{2} \mathrm{O}_{9}$ series, in line with predictions arising from the lanthanide contraction.

The crystal structure of $\mathrm{Lu}_{4} \mathrm{Al}_{2} \mathrm{O}_{9}$ (space group $P 2_{1} / c$ ), determined using $\mathrm{Eu}_{4} \mathrm{Al}_{2} \mathrm{O}_{9}$ (Brandle \& Steinfink, 1969) as the starting model, contains two crystallographically distinct $\mathrm{Al}$ sites, four $\mathrm{Lu}$ sites, and nine $\mathrm{O}$ sites. The two $\mathrm{Al}$ sites are tetrahedrally coordinated by oxygen atoms. The two $\mathrm{Al}$ tetrahedra are connected through a shared $\mathrm{O} 5$ atom, forming an $\mathrm{Al}_{2} \mathrm{O}_{7}$ ditetrahedral oxy-aluminate group (Fig. 2). The $\mathrm{Al}_{2} \mathrm{O}_{7}$ dimers lie parallel to the $a$ axis, and are related by the $c$ glide symmetry operation (Fig. 3). The average Al1-O and 
$\mathrm{Al} 2-\mathrm{O}$ distances in $\mathrm{Lu}_{4} \mathrm{Al}_{2} \mathrm{O}_{9}$ are 1.744 and $1.756 \AA$, respectively, which are comparable to values found in $\mathrm{Eu}_{4} \mathrm{Al}_{2} \mathrm{O}_{9}$ (1.741 and $1.755 \AA$, Brandle \& Steinfink, 1969) and $\mathrm{Y}_{4} \mathrm{Al}_{2} \mathrm{O}_{9}$ (1.739 and $1.769 \AA$, Lehmann et al., 1987). The bondvalence sums (BVS; Brown \& Altermatt, 1985) calculated using the $\mathrm{Al}-\mathrm{O}$ distances and bond-valence parameters recently reported by Gagne \& Hawthorne (2015) $\left(r_{0}=1.634 \AA\right.$, $b=0.39$ ) are 3.02 and 2.93 for Al1 and Al2, respectively. These $\mathrm{BVS}$ values are close to those expected for trivalent $\mathrm{Al}$. The $\mathrm{Al} 1-\mathrm{O} 5-\mathrm{Al} 2$ angle of the $\mathrm{Al}_{2} \mathrm{O}_{7}$ dimer is 134.9 (3) ${ }^{\circ}$, which is smaller than the corresponding angles in $\mathrm{Eu}_{4} \mathrm{Al}_{2} \mathrm{O}_{9}\left(141.9^{\circ}\right.$; Brandle \& Steinfink, 1969) and $\mathrm{Y}_{4} \mathrm{Al}_{2} \mathrm{O}_{9}$ (137.6 ${ }^{\circ}$; Lehmann et al., 1987).

Of the four crystallographically distinct Lu atoms, Lu1 and Lu3 are coordinated by seven oxygen atoms with five $\mathrm{Lu}-\mathrm{O}$ distances in the range $2.219(5)-2.344$ (5) $\AA$ and two in the range 2.461 (6)-2.573 (6) $\AA$. The remaining Lu atoms, Lu 2 and Lu4, are coordinated by six oxygen atoms in distorted octahedra with $\mathrm{Lu}-\mathrm{O}$ distances in the range $2.172(6)-$ 2.337 (6) ̊.

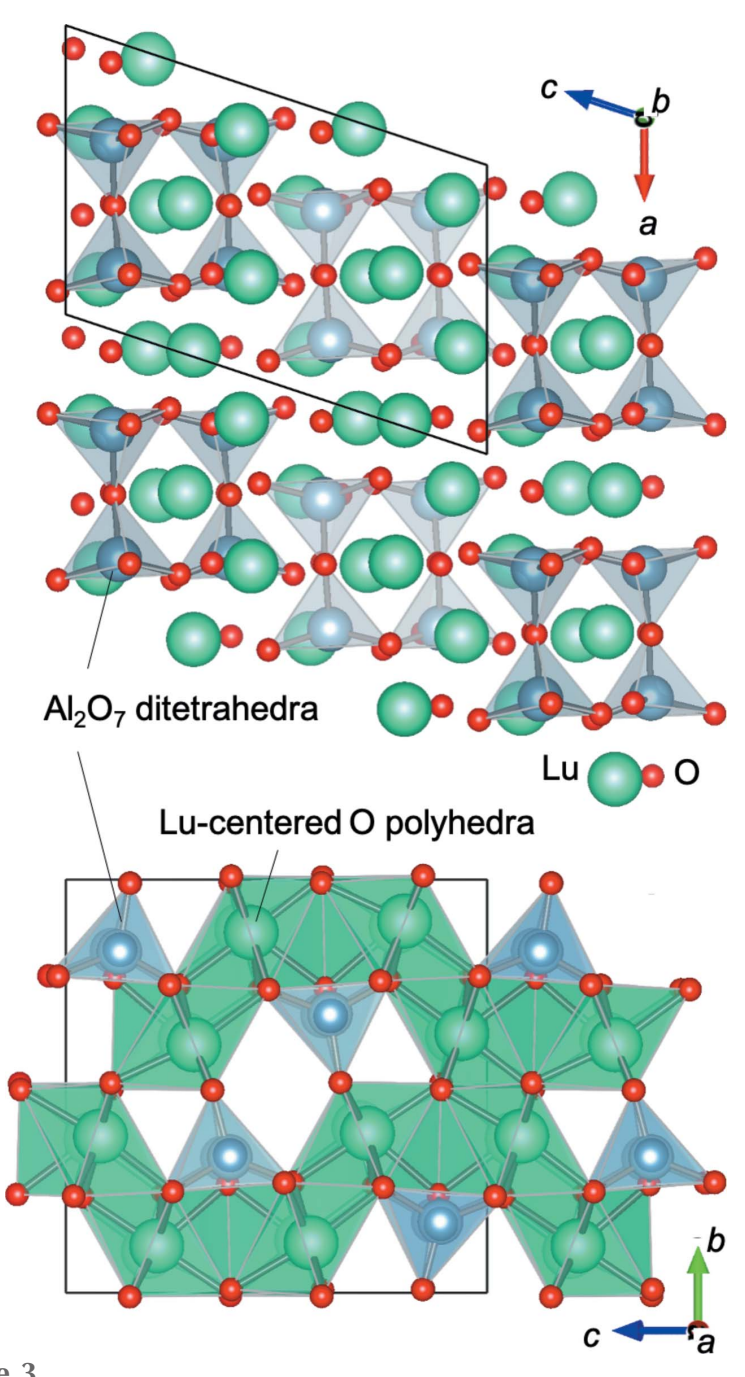

Figure 3

The crystal structure of $\mathrm{Lu}_{4} \mathrm{Al}_{2} \mathrm{O}_{9}$ highlighting the $\mathrm{Al}_{2} \mathrm{O}_{7}$ ditetrahedra viewed down the $b$ axis (upper), and the $\mathrm{Al}_{2} \mathrm{O}_{7}$ ditetrahedra and $\mathrm{Lu}$ centered polyhedra viewed down the $a$ axis (lower).
The averages $\mathrm{Lu}-\mathrm{O}$ distances for the six-fold coordinated Lu atoms in $\mathrm{Lu}_{4} \mathrm{Al}_{2} \mathrm{O}_{9}$ are 2.250 and $2.260 \AA$ for $\mathrm{Lu} 2$ and $\mathrm{Lu} 4$, respectively. These values are $0.02-0.10 \AA$ shorter than those for the $\mathrm{LuO}_{6}$ octahedra found in $\mathrm{Lu}_{3} \mathrm{Al}_{5} \mathrm{O}_{12}$ (2.352 $\AA$; Euler \& Bruce, 1965) and $\mathrm{LuAlO}_{3}$ (2.330 ̊; Shishido et al., 1995).

The average values for the $\mathrm{Eu}-\mathrm{O}$ and $\mathrm{Y}-\mathrm{O}$ distances in $\mathrm{Eu}_{4} \mathrm{Al}_{2} \mathrm{O}_{9}$ and $\mathrm{Y}_{4} \mathrm{Al}_{2} \mathrm{O}_{9}$ lie in the ranges 2.328-2.439 (Brandle \& Steinfink, 1969) and 2.286-2.387 A (Lehmann et al., 1987), respectively. The differences between the $R E-\mathrm{O}$ lengths in $R E_{4} \mathrm{Al}_{2} \mathrm{O}_{9}$ when $R E=\mathrm{Eu}$ and $\mathrm{Lu}(0.07-0.09 \AA)$, and when $R E=\mathrm{Y}$ and $\mathrm{Lu}(0.02-0.05 \AA)$ correspond to the differences between ${ }^{\mathrm{VI}} r_{\mathrm{Eu}}-{ }^{\mathrm{VI}} r_{\mathrm{Lu}}(0.086 \AA)$ and ${ }^{\mathrm{VI}} r_{\mathrm{Y}}-{ }^{\mathrm{VI}} r_{\mathrm{Lu}}$ $(0.039 \AA)$, where ${ }^{\mathrm{VI}} r_{\mathrm{Eu}},{ }^{\mathrm{VI}} r_{\mathrm{Lu}}$, and ${ }^{\mathrm{VI}} r_{\mathrm{Lu}}$ are the effective ionic radii in sixfold coordination of $\mathrm{Lu}^{3+}(0.861 \AA), \mathrm{Eu}^{3+}(0.947 \AA)$, and $\mathrm{Y}^{3+}(0.900 \AA)$, respectively (Shannon, 1976). The BVS for $\mathrm{Lu} 1, \mathrm{Lu} 2, \mathrm{Lu} 3$, and Lu4, calculated using the bond-valence parameters $\left(r_{0}=1.939 \AA, b=0.403\right)$ of Gagné \& Hawthorne (2015), are 2.766, 2.796, 2.642, and 2.714, respectively, which are smaller than the expected valence value of +3 for the $\mathrm{Lu}$ atoms. The polyhedral volumes of $\mathrm{Lu}_{10}\left(18.18 \AA^{3}\right), \mathrm{Lu}_{2} \mathrm{O}_{6}$ $\left(14.29 \AA^{3}\right),{\mathrm{Lu} 3 \mathrm{O}_{7}}_{7}\left(18.56 \AA^{3}\right)$, and $\mathrm{Lu}_{4} \mathrm{O}_{6}\left(14.24 \AA^{3}\right)$ are 1.1$1.7 \AA^{3}$ and $0.5-0.8 \AA^{3}$ smaller than those for $\mathrm{Eu}_{4} \mathrm{Al}_{2} \mathrm{O}_{9}$ $\left(\mathrm{Eu}_{10}: 19.85 \AA^{3}, \quad \mathrm{Eu}_{2} \mathrm{O}_{6}: 15.38 \AA^{3}, \quad \mathrm{Eu}_{3} \mathrm{O}_{7}: 20.14 \AA^{3}\right.$, and $\left.\mathrm{Eu}_{4} \mathrm{O}_{6}: 15.71 \AA^{3}\right)$ and for $\mathrm{Y}_{4} \mathrm{Al}_{2} \mathrm{O}_{9}\left(\mathrm{Y} 1 \mathrm{O}_{7}: 18.66 \AA^{3}, \mathrm{Y}_{2} \mathrm{O}_{6}\right.$ : $14.77 \AA^{3}, \mathrm{Y}_{3} \mathrm{O}_{7}: 19.33 \AA^{3}$, and $\mathrm{Y}_{40}: 14.98 \AA^{3}$ ), respectively. These differences in polyhedral volumes correlate with the differences in ionic radii of the lanthanides.

\section{Synthesis and crystallization}

The starting powders $\mathrm{Al}_{2} \mathrm{O}_{3}$ (Sumitomo Chemicals, AKP20, 99.99\%) and $\mathrm{Lu}_{2} \mathrm{O}_{3}$ (Nippon Yttrium, 99.999\%) were mixed in a molar ratio of $\mathrm{Lu}: \mathrm{Al}=2: 1$, ground with ethanol in an agate mortar, and pressed into a pellet. The pellet was placed in a $\mathrm{BN}$ crucible with an inner diameter of $18 \mathrm{~mm}$ and a height of $20 \mathrm{~mm}$. The $\mathrm{BN}$ crucible was covered with a BN lid, and heated in a chamber with a carbon heater (Shimadzu Mectem, Inc., VESTA). The pellet was heated slowly under vacuum $\left(\sim 10^{-2} \mathrm{~Pa}\right)$ from room temperature to $1273 \mathrm{~K}$. During the $5 \mathrm{~min}$. hold at $1273 \mathrm{~K}$, the chamber was filled with $\mathrm{Ar}$ $(99.9995 \%)$ up to $0.15 \mathrm{MPa}$. The temperature was then raised to $2173 \mathrm{~K}$ at a heating rate of $300 \mathrm{Kh}^{-1}$. After being held at $2173 \mathrm{~K}$ for $4 \mathrm{~h}$, the sample was cooled to $1473 \mathrm{~K}$ at a rate of 20 $\mathrm{Kh}^{-1}$, and then to room temperature by shutting off the heater. A part of the obtained sample was pulverized in the agate mortar, and powder X-ray diffraction measurements (Bruker D2 Phaser, $\mathrm{Cu} K \alpha$ radiation) confirmed that the major crystalline phase present in the sample was $\mathrm{Lu}_{4} \mathrm{Al}_{2} \mathrm{O}_{9}$, together with small amounts of $\mathrm{LuAlO}_{3}$ and unreacted $\mathrm{Lu}_{2} \mathrm{O}_{3}$ (Fig. S1a). Colorless crystals of $\mathrm{Lu}_{4} \mathrm{Al}_{2} \mathrm{O}_{9}$ were selected for single-crystal X-ray diffraction studies.

\section{Refinement}

Crystal data, data collection and structure refinement details are summarized in Table 1. The Eu atoms in the rare-earth metal sites in the structural model of $\mathrm{Eu}_{4} \mathrm{Al}_{2} \mathrm{O}_{9}$ (Brandle \& 
Table 1

Experimental details.

\begin{tabular}{|c|c|}
\hline \multicolumn{2}{|l|}{ Crystal data } \\
\hline Chemical formula & $\mathrm{Lu}_{4} \mathrm{Al}_{2} \mathrm{O}_{9}$ \\
\hline$M_{\mathrm{r}}$ & 897.84 \\
\hline Crystal system, space group & Monoclinic, $P 2_{1} / c$ \\
\hline Temperature $(\mathrm{K})$ & 301 \\
\hline$a, b, c(\AA)$ & $\begin{array}{l}7.2360(11), 10.3330(19) \\
11.096(3)\end{array}$ \\
\hline$\beta\left(^{\circ}\right)$ & $108.381(11)$ \\
\hline$V\left(\AA^{3}\right)$ & $787.3(3)$ \\
\hline$Z$ & 4 \\
\hline Radiation type & Мо $K \alpha$ \\
\hline$\mu\left(\mathrm{mm}^{-1}\right)$ & 49.97 \\
\hline Crystal size (mm) & $0.12 \times 0.05 \times 0.04$ \\
\hline \multicolumn{2}{|l|}{ Data collection } \\
\hline Diffractometer & Bruker D8 QUEST \\
\hline Absorption correction & $\begin{array}{l}\text { Multi-scan (SADABS; Bruker, } \\
\text { 2016) }\end{array}$ \\
\hline$T_{\min }, T_{\max }$ & $0.451,0.746$ \\
\hline $\begin{array}{l}\text { No. of measured, independent and } \\
\text { observed }[I>2 \sigma(I)] \text { reflections }\end{array}$ & $32672,2795,2719$ \\
\hline$R_{\text {int }}$ & 0.035 \\
\hline$(\sin \theta / \lambda)_{\max }\left(\AA^{-1}\right)$ & 0.748 \\
\hline \multicolumn{2}{|l|}{ Refinement } \\
\hline$R\left[F^{2}>2 \sigma\left(F^{2}\right)\right], w R\left(F^{2}\right), S$ & $0.019,0.043,1.17$ \\
\hline No. of reflections & 2795 \\
\hline No. of parameters & 138 \\
\hline$\Delta \rho_{\max }, \Delta \rho_{\min }\left(\mathrm{e} \AA^{-3}\right)$ & $1.49,-1.81$ \\
\hline
\end{tabular}

Computer programs: APEX3 (Bruker, 2018), SAINT (Bruker, 2017), SHELXL2014/7 (Sheldrick, 2015), VESTA (Momma \& Izumi, 2011) and publCIF (Westrip, 2010).

Steinfink, 1969) were replaced by $\mathrm{Lu}$ atoms to generate the initial model. Several iterations of refinement yielded an $R$ value of $10.07 \%$ and a residual electron density of $\sim 10$ e $\AA^{-3}$. A subsequent refinement, performed by implementing the (100) twin plane observed in a study of $\mathrm{Y}_{4} \mathrm{Al}_{2} \mathrm{O}_{9}$ (Yamane et al., 1995b), yielded an $R\left(F^{2}>2 \sigma\left(F^{2}\right)\right)$ value of $1.92 \%$ with an approximate volume ratio of $6: 4$ for the twin domains.

\section{Acknowledgements}

We are grateful to Ms Yuko Suzuki and Ms Mitsuyo Takaishi for their assistance with the high-temperature synthesis.

\section{Funding information}

Funding for this research was provided by the Mitsubishi Chemical Group, Science and Technology Research Center, Inc. (a joint research with Tohoku University and the Mitsubishi Chemical Group, Science and Technology Research Center, Inc. J180002907).

\section{References}

Brandle, C. D. \& Steinfink, H. (1969). Inorg. Chem. 8, 1320-1324. Brown, I. D. \& Altermatt, D. (1985). Acta Cryst. B41, 244-247. Bruker (2016). SADABS. Bruker AXS Inc., Madison, Wisconsin, USA.

Bruker (2017). SAINT. Bruker AXS Inc., Madison, Wisconsin, USA. Bruker (2018). APEX3 . Bruker AXS Inc., Madison, Wisconsin, USA.

Christensen, A. N. \& Hazell, R. G. (1991). Acta Chem. Scand. 45, 226230.
Dernier, P. D. \& Maines, R. G. (1971). Mater. Res. Bull. 6, 433-439.

Ding, L., Zhang, Q., Luo, J., Liu, W., Zhou, W. \& Yin, S. (2011). J. Alloys Compd. 509, 10167-10171.

Dohrup, J., Hoyvald, A., Mogensen, G., Jacobsen, C. J. H. \& Villadsen, J. (1996). J. Am. Ceram. Soc. 79, 2959-2960.

Drozdowski, W., Lukasiewicz, T., Wojtowicz, A. J., Wisniewski, D. \& Kisielewski, J. (2005). J. Cryst. Growth, 275, e709-e714.

Euler, F. \& Bruce, J. A. (1965). Acta Cryst. 19, 971-978.

Gagné, O. C. \& Hawthorne, F. C. (2015). Acta Cryst. B71, 562578.

Jero, P. D. \& Kriven, W. M. (1988). J. Am. Ceram. Soc. 71, C454-C455. Klimm, D. (2010). J. Cryst. Growth, 312, 730-733.

Lehmann, M. S., Christensen, A. N., Fjellvåg, H., Feidenhans'l, R. \& Nielsen, M. (1987). J. Appl. Cryst. 20, 123-129.

Lempicki, A., Brecher, C., Wisniewski, D., Zych, E. \& Wojtowicz, A. J. (1996). IEEE Trans. Nucl. Sci. 43, 1316-1320.

Li, Y. Q., Hirosaki, N., Xie, R. J., Takeda, T., Lofland, S. E. \& Ramanujachary, K. V. (2009). J. Alloys Compd. 484, 943-948.

Martín-Sedeño, M. C., Marrero-López, D., Losilla, E. R., Bruque, S., Núñez, P. \& Aranda, M. A. G. (2006). J. Solid State Chem. 179, 3445-3455.

Mizuno, M. (1979). J. Ceram. Soc. Jpn, 87, 405-412.

Mizuno, M. \& Noguchi, T. (1980). J. Ceram. Soc. Jpn, 88, 322-327.

Mizuno, M., Yamada, T. \& Noguchi, T. (1977a). J. Ceram. Soc. Jpn, 85, 374-379.

Mizuno, M., Yamada, T. \& Noguchi, T. (1977b). J. Ceram. Soc. Jpn, 85, 543-548.

Mizuno, M., Yamada, T. \& Noguchi, T. (1978). J. Ceram. Soc. Jpn, 86, 359-364.

Momma, K. \& Izumi, F. (2011). J. Appl. Cryst. 44, 1272-1276.

Nikl, M. (2000). Phys. Status Solidi A, 178, 595-620.

Nikl, M., Yoshikawa, A., Kamada, K., Nejezchleb, K., Stanek, C. R., Mares, J. A. \& Blazek, K. (2013). Prog. Cryst. Growth Charact. Mater. 59, 47-72.

Petrosyan, A. G., Popova, V. F., Gusarov, V. V., Shirinyan, G. O., Pedrini, C. \& Lecoq, P. (2006). J. Cryst. Growth, 293, 74-77.

Petrosyan, A. G., Popova, V. F., Ugolkov, V. L., Romanov, D. P. \& Ovanesyan, K. L. (2013). J. Cryst. Growth, 377, 178-183.

Reed, J. W. \& Chase, A. B. (1962). Acta Cryst. 15, 812.

Shannon, R. D. (1976). Acta Cryst. A32, 751-767.

Sheldrick, G. M. (2015). Acta Cryst. C71, 3-8.

Shirvinskaya, A. K. \& Popova, V. F. (1977). Dokl. Akad. Nauk SSSR, 233, 1110-1113.

Shishido, T., Nojima, S., Tanaka, M., Horiuchi, H. \& Fukuda, T. (1995). J. Alloys Compd. 227, 175-179.

Talik, E., Guzik, A., Zajdel, P., Lipifska, L., Baran, M. \& Szubka, M. (2016). Mater. Res. Bull. 83, 56-64.

Wang, Z., Zou, J., Zhang, C., Yang, B., Shi, M., Li, Y., Zhou, H., Liu, Y., Li, M. \& Liu, Z. (2018). J. Non-Cryst. Solids, 489, 57-63.

Warshaw, I. \& Roy, R. (1959). J. Am. Ceram. Soc. 42, 434-438.

Westrip, S. P. (2010). J. Appl. Cryst. 43, 920-925.

Wojtowicz, A. J. (1999). Acta Phys. Pol. A, 95, 165-178.

Wojtowicz, A. J., Drozdowski, W., Wisniewski, D., Lefaucheur, J.-L., Galazka, Z., Gou, Z., Lukasiewicz, T. \& Kisielewski, J. (2006). Opt. Mater. 28, 85-93.

Wu, P. \& Pelton, A. D. (1992). J. Alloys Compd. 179, 259-287.

Xiang, R., Liang, X., Li, P., Di, X. \& Xiang, W. (2016). Chem. Eng. J. 306, 858-865.

Yamane, H., Ogawara, K., Omori, M. \& Hirai, T. (1995a). J. Am. Ceram. Soc. 78, 2385-2390.

Yamane, H., Omori, M. \& Hirai, T. (1995b). J. Mater. Sci. Lett. 14, 561-563.

Zhang, L., Madej, C., Pedrini, C., Dujardin, C., Kamenskikh, I., Belsky, A., Shaw, D. A., Mesnard, P. \& Fouassier, C. (1998). J. Electrochem. Soc.: Proceedings of the Sixth International Conference on Luminescent materials 97, 342-351.

Zhang, L., Madej, C., Pédrini, C., Moine, B., Dujardin, C., Petrosyan, A. \& Belsky, A. N. (1997). Chem. Phys. Lett. 268, 408-412. 


\section{supporting information}

Acta Cryst. (2020). E76, 752-755 [https://doi.org/10.1107/S2056989020005757]

\section{Crystal structure of lutetium aluminate (LUAM), $\mathrm{Lu}_{4} \mathrm{Al}_{2} \mathrm{O}_{9}$}

\section{Rayko Simura and Hisanori Yamane}

\section{Computing details}

Data collection: APEX3 (Bruker, 2018); cell refinement: APEX3 (Bruker, 2018); data reduction: SAINT (Bruker, 2017); program(s) used to refine structure: SHELXL2014/7 (Sheldrick, 2015b); molecular graphics: VESTA (Momma \& Izumi, 2011); software used to prepare material for publication: publCIF (Westrip, 2010).

Lutetium aluminate

Crystal data

$\mathrm{Lu}_{4} \mathrm{Al}_{2} \mathrm{O}_{9}$

$M_{r}=897.84$

Monoclinic, $P 2_{1} / c$

$a=7.2360(11) \AA$

$b=10.3330(19) \AA$

$c=11.096(3) \AA$

$\beta=108.381(11)^{\circ}$

$V=787.3(3) \AA^{3}$

$Z=4$

\section{Data collection}

Bruker D8 QUEST diffractometer

Detector resolution: 7.3910 pixels $\mathrm{mm}^{-1}$ $\omega$ and $\sigma$ cans

Absorption correction: multi-scan

(SADABS; Bruker, 2016)

$T_{\min }=0.451, T_{\max }=0.746$

32672 measured reflections

\section{Refinement}

Refinement on $F^{2}$

Least-squares matrix: full

$R\left[F^{2}>2 \sigma\left(F^{2}\right)\right]=0.019$

$w R\left(F^{2}\right)=0.043$

$S=1.17$

2795 reflections

138 parameters

0 restraints
$F(000)=1528$

$D_{\mathrm{x}}=7.575 \mathrm{Mg} \mathrm{m}^{-3}$

Mo $K \alpha$ radiation, $\lambda=0.71073 \AA$

Cell parameters from 1294 reflections

$\theta=2.8-38.5^{\circ}$

$\mu=49.97 \mathrm{~mm}^{-1}$

$T=301 \mathrm{~K}$

Chip, colourless

$0.12 \times 0.05 \times 0.04 \mathrm{~mm}$

2795 independent reflections

2719 reflections with $I>2 \sigma(I)$

$R_{\text {int }}=0.035$

$\theta_{\max }=32.1^{\circ}, \theta_{\min }=2.8^{\circ}$

$h=-10 \rightarrow 10$

$k=-15 \rightarrow 15$

$l=-16 \rightarrow 16$

$$
\begin{aligned}
& w=1 /\left[\sigma^{2}\left(F_{\mathrm{o}}^{2}\right)+17.273 P\right] \\
& \quad \text { where } P=\left(F_{\mathrm{o}}^{2}+2 F_{\mathrm{c}}^{2}\right) / 3 \\
& (\Delta / \sigma)_{\max }=0.001 \\
& \Delta \rho_{\max }=1.49 \mathrm{e} \AA^{-3} \\
& \Delta \rho_{\min }=-1.81 \mathrm{e} \AA^{-3} \\
& \text { Extinction correction: } S H E L X L 2014 / 7 \\
& \quad(\text { Sheldrick } 2015 \mathrm{~b}), \\
& \mathrm{Fc}^{*}=\mathrm{kFc}\left[1+0.001 \mathrm{xFc}^{2} \lambda^{3} / \sin (2 \theta)\right]^{-1 / 4}
\end{aligned}
$$

Extinction coefficient: 0.00026 (2) 


\section{Special details}

Geometry. All esds (except the esd in the dihedral angle between two 1.s. planes) are estimated using the full covariance matrix. The cell esds are taken into account individually in the estimation of esds in distances, angles and torsion angles; correlations between esds in cell parameters are only used when they are defined by crystal symmetry. An approximate (isotropic) treatment of cell esds is used for estimating esds involving l.s. planes.

Refinement. Refined as a two-component inversion twin

Fractional atomic coordinates and isotropic or equivalent isotropic displacement parameters $\left(\hat{A}^{2}\right)$

\begin{tabular}{lllll}
\hline & $x$ & $y$ & $z$ & $U_{\text {iso }} * U_{\text {eq }}$ \\
\hline A11 & $0.2142(4)$ & $0.1742(2)$ & $0.1270(2)$ & $0.0058(4)$ \\
A12 & $0.6551(4)$ & $0.1717(2)$ & $0.1108(2)$ & $0.0059(4)$ \\
Lu1 & $0.52225(7)$ & $0.11375(3)$ & $0.78409(2)$ & $0.00572(6)$ \\
Lu2 & $0.02236(6)$ & $0.10027(3)$ & $0.80405(2)$ & $0.00574(6)$ \\
Lu3 & $0.34172(7)$ & $0.12783(3)$ & $0.44005(2)$ & $0.00605(6)$ \\
Lu4 & $0.83940(6)$ & $0.12082(3)$ & $0.41774(3)$ & $0.00610(6)$ \\
O1 & $0.7934(8)$ & $0.2450(6)$ & $0.7469(5)$ & $0.0102(11)$ \\
O2 & $0.2314(8)$ & $0.2439(5)$ & $0.7699(5)$ & $0.0072(11)$ \\
O3 & $0.2106(13)$ & $0.0095(5)$ & $0.1516(5)$ & $0.0102(10)$ \\
O4 & $0.0720(8)$ & $0.2340(6)$ & $0.9813(6)$ & $0.0092(11)$ \\
O5 & $0.4326(10)$ & $0.2381(4)$ & $0.1156(5)$ & $0.0085(8)$ \\
O6 & $0.6371(8)$ & $0.2328(5)$ & $0.9599(5)$ & $0.0072(11)$ \\
O7 & $0.6926(13)$ & $0.0084(5)$ & $0.1529(5)$ & $0.0111(10)$ \\
O8 & $0.0764(12)$ & $-0.0082(5)$ & $0.3927(5)$ & $0.0072(9)$ \\
O9 & $0.5643(13)$ & $0.0063(5)$ & $0.3906(5)$ & $0.0069(9)$ \\
\hline
\end{tabular}

Atomic displacement parameters $\left(\AA^{2}\right)$

\begin{tabular}{lllllll}
\hline & $U^{11}$ & $U^{22}$ & $U^{33}$ & $U^{12}$ & $U^{13}$ & $U^{23}$ \\
\hline A11 & $0.0071(12)$ & $0.0044(8)$ & $0.0067(9)$ & $0.0012(8)$ & $0.0032(8)$ & $0.0005(7)$ \\
A12 & $0.0074(12)$ & $0.0050(8)$ & $0.0055(8)$ & $0.0010(8)$ & $0.0021(8)$ & $0.0012(7)$ \\
Lu1 & $0.00622(14)$ & $0.00463(12)$ & $0.00594(10)$ & $0.00019(12)$ & $0.00140(14)$ & $-0.00101(8)$ \\
Lu2 & $0.00566(13)$ & $0.00433(12)$ & $0.00733(10)$ & $-0.00031(13)$ & $0.00218(14)$ & $-0.00058(8)$ \\
Lu3 & $0.00647(13)$ & $0.00518(11)$ & $0.00625(10)$ & $0.00024(13)$ & $0.00165(13)$ & $0.00101(9)$ \\
Lu4 & $0.00538(14)$ & $0.00435(10)$ & $0.00863(10)$ & $0.00048(13)$ & $0.00231(15)$ & $0.00137(9)$ \\
O1 & $0.008(3)$ & $0.013(3)$ & $0.008(2)$ & $0.0039(19)$ & $0.0002(18)$ & $0.002(2)$ \\
O2 & $0.009(3)$ & $0.006(2)$ & $0.007(2)$ & $0.0012(18)$ & $0.0030(19)$ & $0.0010(17)$ \\
O3 & $0.011(3)$ & $0.006(2)$ & $0.015(2)$ & $0.000(3)$ & $0.007(3)$ & $0.0006(17)$ \\
O4 & $0.009(3)$ & $0.007(2)$ & $0.009(2)$ & $0.0039(18)$ & $-0.0009(18)$ & $-0.0003(19)$ \\
O5 & $0.008(2)$ & $0.0064(18)$ & $0.012(2)$ & $-0.003(2)$ & $0.004(2)$ & $-0.0003(15)$ \\
O6 & $0.008(3)$ & $0.007(2)$ & $0.007(2)$ & $0.0017(18)$ & $0.0018(18)$ & $0.0009(17)$ \\
O7 & $0.013(3)$ & $0.008(2)$ & $0.016(2)$ & $0.003(3)$ & $0.009(3)$ & $0.0059(18)$ \\
O8 & $0.005(3)$ & $0.006(2)$ & $0.009(2)$ & $-0.003(2)$ & $0.000(3)$ & $-0.0002(16)$ \\
O9 & $0.010(3)$ & $0.0042(19)$ & $0.007(2)$ & $0.001(3)$ & $0.003(3)$ & $0.0001(16)$ \\
& & & & & & \\
\hline
\end{tabular}


Geometric parameters $\left(\AA,{ }^{o}\right)$

\begin{tabular}{|c|c|c|c|}
\hline $\mathrm{A} 11-\mathrm{O} 3$ & $1.724(6)$ & $\mathrm{Lu} 3-\mathrm{O}^{\text {iv }}$ & $2.310(5)$ \\
\hline $\mathrm{A} 11-\mathrm{O} 4^{\mathrm{i}}$ & $1.733(6)$ & $\mathrm{Lu} 3-\mathrm{O}^{\mathrm{ii}}$ & $2.529(5)$ \\
\hline $\mathrm{A} 11-\mathrm{O} 5$ & $1.754(7)$ & $\mathrm{Lu} 3-\mathrm{O} 4^{\mathrm{ii}}$ & $2.573(6)$ \\
\hline $\mathrm{A} 11-\mathrm{O} 2^{\mathrm{ii}}$ & $1.767(6)$ & $\mathrm{Lu} 3-\mathrm{A} 12^{\mathrm{iv}}$ & $3.211(2)$ \\
\hline All-Lu1 $1^{\mathrm{ii}}$ & $3.219(2)$ & $\mathrm{Lu} 3-\mathrm{A} 11^{\mathrm{iv}}$ & $3.247(2)$ \\
\hline $\mathrm{All}-\mathrm{Lu}^{\mathrm{ii}}$ & $3.247(2)$ & Lu3-Lu3 $3^{\mathrm{iii}}$ & $3.4748(8)$ \\
\hline Al1-Lu3 & $3.336(2)$ & $\mathrm{Lu} 3-\mathrm{Lu} 4^{\mathrm{iii}}$ & $3.4803(7)$ \\
\hline $\mathrm{A} 12-\mathrm{O} 7$ & $1.749(6)$ & $\mathrm{Lu} 4-O 4^{\text {ix }}$ & $2.198(6)$ \\
\hline $\mathrm{A} 12-\mathrm{O} 1^{\mathrm{ii}}$ & $1.753(6)$ & $\mathrm{Lu} 4-\mathrm{O} 9$ & $2.253(8)$ \\
\hline $\mathrm{A} 12-\mathrm{O}^{\mathrm{i}}$ & $1.755(6)$ & $\mathrm{Lu} 4-\mathrm{O}^{\mathrm{ii}}$ & $2.255(6)$ \\
\hline $\mathrm{A} 12-\mathrm{O} 5$ & $1.767(7)$ & $\mathrm{Lu} 4-\mathrm{O}^{\mathrm{v}}$ & $2.257(8)$ \\
\hline $\mathrm{A} 12-\mathrm{Lu}^{\mathrm{ii}}$ & $3.211(2)$ & $\mathrm{Lu} 4-\mathrm{O} 1^{\mathrm{ii}}$ & $2.287(6)$ \\
\hline $\mathrm{A} 12-\mathrm{Lu} 1^{\mathrm{ii}}$ & $3.272(2)$ & $\mathrm{Lu} 4-\mathrm{O} 8^{\mathrm{iii}}$ & $2.311(5)$ \\
\hline $\mathrm{A} 12-\mathrm{Lu} 4$ & $3.285(2)$ & $\mathrm{Lu} 4-\mathrm{Lu} 3^{\mathrm{iii}}$ & $3.4804(7)$ \\
\hline $\mathrm{Lu} 1-\mathrm{O} 9^{\mathrm{iii}}$ & $2.219(5)$ & $\mathrm{Lu} 4-\mathrm{Lu} 4^{\mathrm{x}}$ & $3.5061(8)$ \\
\hline $\mathrm{Lu} 1-\mathrm{O} 6$ & $2.233(5)$ & $\mathrm{Lu} 4-\mathrm{Lu} 2^{\mathrm{ix}}$ & $3.5641(7)$ \\
\hline $\mathrm{Lu} 1-\mathrm{O} 3^{\mathrm{iii}}$ & $2.236(8)$ & $\mathrm{Lu} 4-\mathrm{Lu}^{\mathrm{v}}$ & $3.5652(7)$ \\
\hline $\mathrm{Lu} 1-\mathrm{O} 7^{\mathrm{iii}}$ & $2.277(8)$ & $\mathrm{Lu} 4-\mathrm{Lu} 1^{\mathrm{ii}}$ & $3.5836(7)$ \\
\hline $\mathrm{Lu} 1-\mathrm{O} 5^{\mathrm{iv}}$ & $2.344(5)$ & $\mathrm{O} 1-\mathrm{A} 12^{\mathrm{iv}}$ & $1.753(6)$ \\
\hline $\mathrm{Lu} 1-\mathrm{O} 2$ & $2.461(6)$ & $\mathrm{O} 1-\mathrm{Lu} 2^{\mathrm{v}}$ & $2.172(6)$ \\
\hline Lu1-O1 & $2.524(6)$ & $\mathrm{O} 1-\mathrm{Lu} 4^{\mathrm{iv}}$ & $2.287(6)$ \\
\hline $\mathrm{Lu} 1-\mathrm{A} 11^{\mathrm{iv}}$ & $3.219(2)$ & $\mathrm{O} 2-\mathrm{A} 11^{\mathrm{iv}}$ & $1.767(6)$ \\
\hline $\mathrm{Lu} 1-\mathrm{A} 12^{\mathrm{iv}}$ & $3.272(2)$ & $\mathrm{O} 2-\mathrm{Lu}^{3 \mathrm{iv}}$ & $2.238(5)$ \\
\hline $\mathrm{Lu} 1-\mathrm{Lu}^{\mathrm{v}}$ & $3.5579(7)$ & $\mathrm{O} 3-\mathrm{Lu} 2^{\mathrm{vii}}$ & $2.213(8)$ \\
\hline $\mathrm{Lu} 1-\mathrm{Lu} 4^{\mathrm{iv}}$ & $3.5836(7)$ & $\mathrm{O} 3-\mathrm{Lu} 1^{\mathrm{iii}}$ & $2.236(8)$ \\
\hline Lu1-Lu3 & $3.6270(9)$ & $\mathrm{O} 4-\mathrm{Al} 1^{\mathrm{xi}}$ & $1.733(6)$ \\
\hline $\mathrm{Lu} 2-\mathrm{O} 1^{\mathrm{vi}}$ & $2.172(6)$ & $\mathrm{O} 4-\mathrm{Lu} 4^{\mathrm{viii}}$ & $2.198(6)$ \\
\hline $\mathrm{Lu} 2-\mathrm{O}^{\mathrm{vii}}$ & $2.213(8)$ & $\mathrm{O} 4-\mathrm{Lu}^{\mathrm{iv}}$ & $2.573(6)$ \\
\hline $\mathrm{Lu} 2-\mathrm{O} 2$ & $2.235(6)$ & $\mathrm{O} 5-\mathrm{Lu}^{3 \mathrm{ii}}$ & $2.310(5)$ \\
\hline $\mathrm{Lu} 2-\mathrm{O} 7^{\mathrm{iii}}$ & $2.263(8)$ & $\mathrm{O} 5-\mathrm{Lu} 1^{\mathrm{ii}}$ & $2.344(5)$ \\
\hline $\mathrm{Lu} 2-\mathrm{O}^{\mathrm{vii}}$ & $2.280(5)$ & $\mathrm{O} 6-\mathrm{Al}^{\mathrm{xi}}$ & $1.754(6)$ \\
\hline $\mathrm{Lu} 2-\mathrm{O} 4$ & $2.337(6)$ & O6-Lu4 ${ }^{\text {iv }}$ & $2.255(6)$ \\
\hline $\mathrm{Lu} 2-\mathrm{Lu} 1^{\mathrm{vi}}$ & $3.5579(7)$ & O6-Lu3 ${ }^{\text {iv }}$ & $2.529(5)$ \\
\hline Lu2-Lu4 $4^{\text {viii }}$ & $3.5641(7)$ & $\mathrm{O} 7-\mathrm{Lu} 2^{\mathrm{iii}}$ & $2.263(8)$ \\
\hline $\mathrm{Lu} 2-\mathrm{Lu}^{3 \mathrm{iv}}$ & $3.6485(7)$ & $\mathrm{O} 7-\mathrm{Lu} 1^{\mathrm{iii}}$ & $2.277(8)$ \\
\hline $\mathrm{Lu} 2-\mathrm{Lu} 4^{\mathrm{iii}}$ & $3.7187(7)$ & $\mathrm{O} 8-\mathrm{Lu} 4^{\mathrm{vi}}$ & $2.257(8)$ \\
\hline $\mathrm{Lu} 2-\mathrm{Lu}^{\mathrm{vii}}$ & $3.9156(7)$ & $\mathrm{O} 8-\mathrm{Lu} 2^{\mathrm{vii}}$ & $2.280(5)$ \\
\hline $\mathrm{Lu} 3-\mathrm{O} 2^{\mathrm{ii}}$ & $2.238(5)$ & $\mathrm{O} 8-\mathrm{Lu} 4^{\mathrm{iii}}$ & $2.311(5)$ \\
\hline Lu3-O9 & $2.242(8)$ & O9-Lu $1^{i i i}$ & $2.219(5)$ \\
\hline $\mathrm{Lu} 3-\mathrm{O} 9^{\mathrm{iii}}$ & $2.260(5)$ & O9-Lu3 ${ }^{\text {iii }}$ & $2.260(5)$ \\
\hline $\mathrm{Lu} 3-\mathrm{O} 8$ & $2.302(7)$ & & \\
\hline $\mathrm{O} 3-\mathrm{A} 11-\mathrm{O} 4^{\mathrm{i}}$ & $117.7(3)$ & $\mathrm{O} 2^{\mathrm{ii}-\mathrm{Lu} 3-\mathrm{O} 8}$ & $96.84(19)$ \\
\hline $\mathrm{O} 3-\mathrm{A} 11-\mathrm{O} 5$ & $116.2(4)$ & $\mathrm{O} 9-\mathrm{Lu} 3-\mathrm{O} 8$ & $102.35(18)$ \\
\hline $\mathrm{O} 4 \mathrm{i}-\mathrm{A} 11-\mathrm{O} 5$ & $94.7(3)$ & $\mathrm{O} 9^{\mathrm{iii}-\mathrm{Lu} 3-\mathrm{O} 8}$ & $80.0(2)$ \\
\hline $\mathrm{O} 3-\mathrm{A} 11-\mathrm{O}^{\mathrm{ii}}$ & $109.3(3)$ & $\mathrm{O} 2^{\mathrm{ii}}-\mathrm{Lu} 3-\mathrm{O}^{\mathrm{iv}}$ & $106.69(18)$ \\
\hline
\end{tabular}




\begin{tabular}{|c|c|c|c|}
\hline $\mathrm{O} 4^{\mathrm{i}}-\mathrm{A} 11-\mathrm{O} 2^{\mathrm{ii}}$ & $121.3(3)$ & $\mathrm{O} 9-\mathrm{Lu} 3-\mathrm{O}^{\mathrm{iv}}$ & $120.4(2)$ \\
\hline $\mathrm{O} 5-\mathrm{A} 11-\mathrm{O} 2^{\mathrm{ii}}$ & $94.3(3)$ & 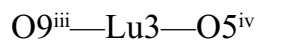 & $74.68(16)$ \\
\hline $\mathrm{O} 3-\mathrm{A} 11-\mathrm{Lu} 1^{\mathrm{ii}}$ & $128.9(3)$ & $\mathrm{O} 8-\mathrm{Lu} 3-\mathrm{O}^{\mathrm{iv}}$ & $123.6(2)$ \\
\hline $\mathrm{O} 4^{\mathrm{i}}-\mathrm{A} 11-\mathrm{Lu} 1^{\mathrm{ii}}$ & $111.6(2)$ & 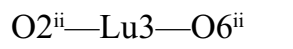 & $78.65(19)$ \\
\hline $\mathrm{O} 5-\mathrm{A} 11-\mathrm{Lu} 1^{\mathrm{ii}}$ & $45.30(17)$ & $\mathrm{O} 9-\mathrm{Lu} 3-\mathrm{O}^{\mathrm{ii}}$ & $71.8(2)$ \\
\hline $\mathrm{O} 2^{\mathrm{ii}}-\mathrm{A} 11-\mathrm{Lu} 1^{\mathrm{ii}}$ & $49.24(19)$ & $\mathrm{O} 9^{\mathrm{iii}}-\mathrm{Lu} 3-\mathrm{O} 6^{\mathrm{ii}}$ & $104.6(2)$ \\
\hline $\mathrm{O} 3-\mathrm{A} 11-\mathrm{Lu}^{\mathrm{ii}}$ & $138.1(3)$ & $\mathrm{O} 8-\mathrm{Lu} 3-\mathrm{O}^{\mathrm{ii}}$ & $171.4(2)$ \\
\hline $\mathrm{O} 4^{\mathrm{i}}-\mathrm{A} 11-\mathrm{Lu} 3^{\mathrm{ii}}$ & $52.0(2)$ & $\mathrm{O} 5^{\mathrm{iv}}-\mathrm{Lu} 3-\mathrm{O} 6^{\mathrm{ii}}$ & $65.0(2)$ \\
\hline $\mathrm{O} 5-\mathrm{A} 11-\mathrm{Lu}^{\mathrm{ii}}$ & $43.34(18)$ & 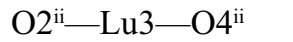 & 74.48 (19) \\
\hline $\mathrm{O} 2^{\mathrm{ii}}-\mathrm{A} 11-\mathrm{Lu} 3^{\mathrm{ii}}$ & $108.5(2)$ & $\mathrm{O} 9-\mathrm{Lu} 3-\mathrm{O} 4^{\mathrm{ii}}$ & $176.2(2)$ \\
\hline $\mathrm{Lu} 1^{\mathrm{ii}}-\mathrm{A} 11-\mathrm{Lu} 3^{\mathrm{ii}}$ & $68.24(5)$ & 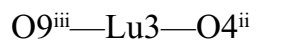 & $103.8(2)$ \\
\hline $\mathrm{O} 3-\mathrm{A} 11-\mathrm{Lu} 3$ & $72.9(2)$ & $\mathrm{O} 8-\mathrm{Lu} 3-\mathrm{O} 4^{\mathrm{ii}}$ & $75.8(2)$ \\
\hline $\mathrm{O} 4 \mathrm{i}-\mathrm{A} 11-\mathrm{Lu} 3$ & $155.5(2)$ & $\mathrm{O}^{\mathrm{iv}}-\mathrm{Lu} 3-\mathrm{O} 4^{\mathrm{ii}}$ & $63.1(2)$ \\
\hline $\mathrm{O} 5-\mathrm{A} 11-\mathrm{Lu} 3$ & 99.75 (19) & 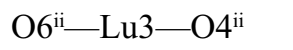 & $109.64(15)$ \\
\hline $\mathrm{O} 2^{\mathrm{ii}}-\mathrm{A} 11-\mathrm{Lu} 3$ & $38.37(18)$ & $\mathrm{O} 2^{\mathrm{ii}}-\mathrm{Lu} 3-\mathrm{A} 12^{\mathrm{iv}}$ & $96.50(15)$ \\
\hline $\mathrm{Lu} 1^{\mathrm{ii}-\mathrm{A} 11-\mathrm{Lu} 3}$ & $67.47(5)$ & $\mathrm{O} 9-\mathrm{Lu} 3-\mathrm{A} 12^{\mathrm{iv}}$ & $94.44(18)$ \\
\hline $\mathrm{Lu}^{\mathrm{ii}}{ }^{\mathrm{i}} \mathrm{A} 11-\mathrm{Lu} 3$ & $135.61(8)$ & 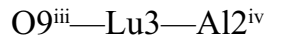 & $86.21(17)$ \\
\hline $\mathrm{O} 7-\mathrm{A} 12-\mathrm{O}^{\mathrm{ii}}$ & $104.2(3)$ & $\mathrm{O} 8-\mathrm{Lu} 3-\mathrm{A} 12^{\mathrm{iv}}$ & $155.68(16)$ \\
\hline $\mathrm{O} 7-\mathrm{A} 12-\mathrm{O}^{\mathrm{i}}$ & $124.1(3)$ & $\mathrm{O} 5^{\mathrm{iv}}-\mathrm{Lu} 3-\mathrm{A} 12^{\mathrm{iv}}$ & $32.39(17)$ \\
\hline $\mathrm{O} 1^{\mathrm{ii}}-\mathrm{A} 12-\mathrm{O}^{\mathrm{i}}$ & $119.7(3)$ & $\mathrm{O} 6^{\mathrm{ii}}-\mathrm{Lu} 3-\mathrm{A} 12^{\mathrm{iv}}$ & $32.95(13)$ \\
\hline $\mathrm{O} 7-\mathrm{A} 12-\mathrm{O} 5$ & $115.6(4)$ & 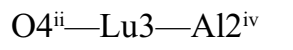 & $88.36(14)$ \\
\hline $\mathrm{O} 1{ }^{\mathrm{ii}}-\mathrm{A} 12-\mathrm{O} 5$ & $93.5(3)$ & $\mathrm{O} 2^{\mathrm{ii}}-\mathrm{Lu} 3-\mathrm{A} 11^{\mathrm{iv}}$ & $93.90(15)$ \\
\hline $\mathrm{O} 6-\mathrm{A} 12-\mathrm{O} 5$ & $95.4(3)$ & O9-Lu3-Al1 ${ }^{\text {iv }}$ & $151.60(17)$ \\
\hline $\mathrm{O} 7-\mathrm{A} 12-\mathrm{Lu} 3^{\mathrm{ii}}$ & $143.2(3)$ & $\mathrm{O} 9^{\mathrm{iii}}-\mathrm{Lu} 3-\mathrm{A} 11^{\mathrm{iv}}$ & $85.82(16)$ \\
\hline 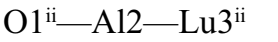 & $107.2(2)$ & O8-Lu3-Al1 ${ }^{\text {iv }}$ & $98.38(18)$ \\
\hline $\mathrm{O} 6^{\mathrm{i}}-\mathrm{A} 12-\mathrm{Lu} 3^{\mathrm{ii}}$ & $51.62(19)$ & $\mathrm{O}^{\mathrm{iv}}-\mathrm{Lu} 3-\mathrm{A} 11^{\mathrm{iv}}$ & $31.41(17)$ \\
\hline $\mathrm{O} 5-\mathrm{A} 12-\mathrm{Lu}^{\mathrm{ii}}$ & $44.47(16)$ & 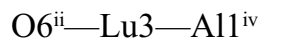 & $89.32(13)$ \\
\hline $\mathrm{O} 7-\mathrm{A} 12-\mathrm{Lu} 1^{\mathrm{ii}}$ & $123.1(2)$ & $\mathrm{O} 4^{\mathrm{ii}}-\mathrm{Lu} 3-\mathrm{All}^{\mathrm{iv}}$ & 32.07 (14) \\
\hline $\mathrm{O} 1^{\mathrm{ii}}-\mathrm{A} 12-\mathrm{Lu} 1^{\mathrm{ii}}$ & $49.8(2)$ & $\mathrm{Al} 2^{\mathrm{iv}}-\mathrm{Lu} 3-\mathrm{Al} 1^{\mathrm{iv}}$ & $60.46(5)$ \\
\hline $\mathrm{O} 6{ }^{\mathrm{i}}-\mathrm{A} 12-\mathrm{Lu} 1^{\mathrm{ii}}$ & $111.7(2)$ & 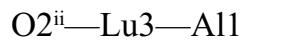 & $29.36(15)$ \\
\hline $\mathrm{O} 5-\mathrm{A} 12-\mathrm{Lu} 1^{\mathrm{ii}}$ & $43.89(17)$ & O9-Lu3-Al1 & $79.05(14)$ \\
\hline $\mathrm{Lu} 3^{3 i}-\mathrm{A} 12-\mathrm{Lu} 1^{\mathrm{ii}}$ & $68.03(5)$ & O9 ${ }^{\mathrm{iii}}-\mathrm{Lu} 3-\mathrm{A} 11$ & $149.97(14)$ \\
\hline $\mathrm{O} 7-\mathrm{A} 12-\mathrm{Lu} 4$ & $65.8(2)$ & O8-Lu3-Al1 & $85.02(13)$ \\
\hline $\mathrm{O} 1^{\mathrm{ii}}-\mathrm{A} 12-\mathrm{Lu} 4$ & $41.4(2)$ & $\mathrm{O} 5^{\mathrm{iv}}-\mathrm{Lu} 3-\mathrm{A} 11$ & $134.72(12)$ \\
\hline $\mathrm{O} 6$ - & $158.5(2)$ & $\mathrm{O} 6^{\mathrm{ii}}-\mathrm{Lu} 3-\mathrm{A} 11$ & $87.58(13)$ \\
\hline $\mathrm{O} 5-\mathrm{A} 12-\mathrm{Lu} 4$ & $96.12(18)$ & 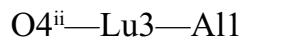 & $97.42(14)$ \\
\hline $\mathrm{Lu} 3^{\mathrm{ii}}-\mathrm{A} 12-\mathrm{Lu} 4$ & $134.03(8)$ & $\mathrm{A} 12^{\mathrm{iv}}-\mathrm{Lu} 3-\mathrm{A} 11$ & $115.70(7)$ \\
\hline $\mathrm{Lu} 1{ }^{\mathrm{ii}}-\mathrm{A} 12-\mathrm{Lu} 4$ & $66.26(5)$ & $\mathrm{A} 11^{\mathrm{iv}}-\mathrm{Lu} 3-\mathrm{A} 11$ & $122.25(5)$ \\
\hline $\mathrm{O} 9^{\mathrm{iii}}-\mathrm{Lu} 1-\mathrm{O} 6$ & $174.8(3)$ & $\mathrm{O} 2^{\mathrm{ii}}-\mathrm{Lu} 3-\mathrm{Lu} 3^{\mathrm{iii}}$ & $142.19(14)$ \\
\hline $\mathrm{O} 9^{i i i-L u 1-O 3^{i i i}}$ & $86.4(2)$ & O9-Lu3-Lu3 ${ }^{\mathrm{iii}}$ & 39.67 (13) \\
\hline $\mathrm{O} 6-\mathrm{Lu} 1-\mathrm{O} 3^{\mathrm{iii}}$ & $89.4(2)$ & $\mathrm{O} 9^{\mathrm{iii}}-\mathrm{Lu} 3-\mathrm{Lu} 3^{\mathrm{iii}}$ & $39.30(19)$ \\
\hline $\mathrm{O} 9^{i i i}-\mathrm{Lu} 1-\mathrm{O} 7^{\mathrm{iii}}$ & $85.8(2)$ & $\mathrm{O} 8-\mathrm{Lu} 3-\mathrm{Lu} 3^{\mathrm{iii}}$ & $91.43(16)$ \\
\hline $\mathrm{O} 6-\mathrm{Lu} 1-\mathrm{O} 7^{\mathrm{iii}}$ & $98.0(2)$ & $\mathrm{O} 5^{\mathrm{iv}}-\mathrm{Lu} 3-\mathrm{Lu} 3^{\mathrm{iii}}$ & $98.91(14)$ \\
\hline 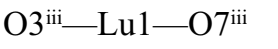 & $101.03(18)$ & $\mathrm{O} 6^{\mathrm{ii}}-\mathrm{Lu} 3-\mathrm{Lu} 3^{\mathrm{iii}}$ & $87.86(13)$ \\
\hline 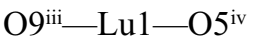 & $74.76(17)$ & $\mathrm{O} 4^{\mathrm{ii}}-\mathrm{Lu} 3-\mathrm{Lu} 3^{\mathrm{iii}}$ & $143.06(13)$ \\
\hline $\mathrm{O} 6-\mathrm{Lu} 1-\mathrm{O} 5^{\mathrm{iv}}$ & $105.69(19)$ & $\mathrm{A} 12^{\mathrm{iv}}-\mathrm{Lu} 3-\mathrm{Lu} 3^{\mathrm{iii}}$ & $90.40(5)$ \\
\hline 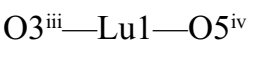 & $128.4(2)$ & $\mathrm{A} 11^{\mathrm{iv}}-\mathrm{Lu} 3-\mathrm{Lu} 3^{\mathrm{iii}}$ & $121.35(4)$ \\
\hline 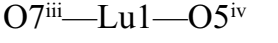 & $124.3(2)$ & Al1-Lu3-Lu3 ${ }^{\mathrm{iii}}$ & $116.13(4)$ \\
\hline
\end{tabular}




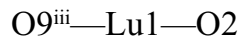

$$
\begin{aligned}
& \text { O6- } \mathrm{Lu} 1-\mathrm{O} 2 \\
& \mathrm{O} 3{ }^{\mathrm{iii}-\mathrm{Lu} 1-\mathrm{O} 2} \\
& \mathrm{O} 7 \mathrm{iii}-\mathrm{Lu} 1-\mathrm{O} 2 \\
& \mathrm{O}^{\mathrm{iv}}-\mathrm{Lu} 1-\mathrm{O} 2
\end{aligned}
$$

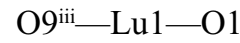

$$
\begin{aligned}
& \text { O6-Lu1-O1 }
\end{aligned}
$$

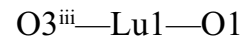

$$
\begin{aligned}
& \text { O7 } 7 \text { iii-Lu1-O1 } \\
& \mathrm{O}^{\mathrm{iv}}-\mathrm{Lu} 1-\mathrm{O} 1 \\
& \mathrm{O} 2-\mathrm{Lu} 1-\mathrm{O} 1 \\
& \text { O9iii-Lu1-Al1 }{ }^{\text {iv }} \\
& \text { O6-Lu1-A11 }{ }^{\text {iv }}
\end{aligned}
$$

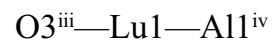

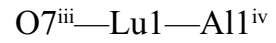

$$
\begin{aligned}
& \mathrm{O}^{\mathrm{iv}}-\mathrm{Lu} 1-\mathrm{Al1}{ }^{\mathrm{iv}} \\
& \mathrm{O} 2-\mathrm{Lu} 1-\mathrm{A} 11^{\mathrm{iv}} \\
& \text { O1-Lu1-Al1 }{ }^{\text {iv }} \\
& \text { O9iii-Lu1- }{ }^{i i 2} 2^{\text {iv }} \\
& \mathrm{O} 6-\mathrm{Lu} 1-\mathrm{A} 12^{\mathrm{iv}}
\end{aligned}
$$

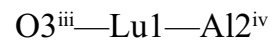

$$
\begin{aligned}
& \mathrm{O} 7^{\mathrm{iii}}-\mathrm{Lu} 1-\mathrm{A} 12^{\mathrm{iv}} \\
& \mathrm{O} 5^{\mathrm{iv}}-\mathrm{Lu} 1-\mathrm{A} 12^{\mathrm{iv}} \\
& \mathrm{O} 2-\mathrm{Lu} 1-\mathrm{A} 12^{\mathrm{iv}} \\
& \mathrm{O} 1-\mathrm{Lu} 1-\mathrm{A} 12^{\mathrm{iv}} \\
& \mathrm{A} 11^{\mathrm{iv}}-\mathrm{Lu} 1-\mathrm{A} 12^{\mathrm{iv}} \\
& \mathrm{O} 9^{\mathrm{iii}}-\mathrm{Lu} 1-\mathrm{Lu} 2^{\mathrm{v}} \\
& \mathrm{O} 6-\mathrm{Lu} 1-\mathrm{Lu} 2^{\mathrm{v}}
\end{aligned}
$$

\begin{tabular}{|c|c|}
\hline $104.5(2)$ & $\mathrm{O} 2^{\mathrm{ii}}-\mathrm{Lu} 3-\mathrm{Lu} 4^{\mathrm{iii}}$ \\
\hline 80.21 (19) & O9-Lu3-Lu4 $4^{\text {iii }}$ \\
\hline $165.4(2)$ & 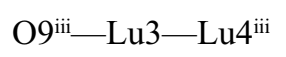 \\
\hline $70.7(2)$ & $\mathrm{O} 8-\mathrm{Lu} 3-\mathrm{Lu} 4^{\mathrm{iii}}$ \\
\hline $64.9(2)$ & $\mathrm{O} 5^{\mathrm{iv}}-\mathrm{Lu} 3-\mathrm{Lu} 4^{\mathrm{iii}}$ \\
\hline $100.2(2)$ & $\mathrm{O} 6^{\mathrm{ii}}-\mathrm{Lu} 3-\mathrm{Lu} 4^{\mathrm{iii}}$ \\
\hline 75.68 (19) & $\mathrm{O} 4^{\mathrm{ii}}-\mathrm{Lu} 3-\mathrm{Lu} 4^{\mathrm{iii}}$ \\
\hline $73.7(2)$ & $\mathrm{A} 12^{\mathrm{iv}}-\mathrm{Lu} 3-\mathrm{Lu} 4^{\mathrm{iii}}$ \\
\hline $171.6(2)$ & $\mathrm{A} 11^{\mathrm{iv}}-\mathrm{Lu} 3-\mathrm{Lu} 4^{\mathrm{iii}}$ \\
\hline $63.4(2)$ & Al1-Lu3-Lu $4^{\text {iii }}$ \\
\hline $112.92(16)$ & $\mathrm{Lu} 3^{\mathrm{iii}}-\mathrm{Lu} 3-\mathrm{Lu} 4^{\mathrm{iii}}$ \\
\hline 87.18 (17) & $\mathrm{O} 4^{\mathrm{ix}}-\mathrm{Lu} 4-\mathrm{O} 9$ \\
\hline $95.83(15)$ & $\mathrm{O} 4^{\mathrm{ix}}-\mathrm{Lu} 4-\mathrm{O} 6^{\mathrm{ii}}$ \\
\hline $160.44(18)$ & $\mathrm{O} 9-\mathrm{Lu} 4-\mathrm{O} 6^{\mathrm{ii}}$ \\
\hline $96.91(18)$ & $\mathrm{O} 4^{\mathrm{ix}}-\mathrm{Lu} 4-\mathrm{O} 8^{\mathrm{v}}$ \\
\hline 32.14 (17) & $\mathrm{O} 9-\mathrm{Lu} 4-\mathrm{O} 8^{\mathrm{v}}$ \\
\hline $32.95(13)$ & $\mathrm{O} 6^{\mathrm{ii}}-\mathrm{Lu} 4-\mathrm{O}^{\mathrm{v}}$ \\
\hline $89.26(14)$ & $\mathrm{O} 4^{\mathrm{ix}}-\mathrm{Lu} 4-\mathrm{O} 1^{\mathrm{ii}}$ \\
\hline $85.38(16)$ & $\mathrm{O} 9-\mathrm{Lu} 4-\mathrm{O} 1^{\mathrm{ii}}$ \\
\hline $92.47(15)$ & $\mathrm{O} 6^{\mathrm{ii}}-\mathrm{Lu} 4-\mathrm{O} 1^{\mathrm{ii}}$ \\
\hline $100.91(19)$ & $\mathrm{O} 8^{\mathrm{v}}-\mathrm{Lu} 4-\mathrm{O} 1^{\mathrm{ii}}$ \\
\hline $155.76(18)$ & $\mathrm{O} 4^{\mathrm{ix}}-\mathrm{Lu} 4-\mathrm{O} 8^{\mathrm{iii}}$ \\
\hline $31.50(17)$ & $\mathrm{O} 9-\mathrm{Lu} 4-\mathrm{O}^{\mathrm{iii}}$ \\
\hline 89.77 (14) & $\mathrm{O} 6^{\mathrm{ii}}-\mathrm{Lu} 4-\mathrm{O} 8^{\mathrm{iii}}$ \\
\hline $32.02(14)$ & $\mathrm{O} 8^{\mathrm{v}}-\mathrm{Lu} 4-\mathrm{O}^{\mathrm{iii}}$ \\
\hline $60.13(5)$ & $\mathrm{O} 1^{\mathrm{ii}}-\mathrm{Lu} 4-\mathrm{O}^{\mathrm{iii}}$ \\
\hline $91.9(2)$ & $\mathrm{O} 4^{\mathrm{ix}}-\mathrm{Lu} 4-\mathrm{Al} 2$ \\
\hline 82.89 (14) & $\mathrm{O} 9-\mathrm{Lu} 4-\mathrm{A} 12$ \\
\hline $36.67(18)$ & $\mathrm{O} 6^{\mathrm{ii}}-\mathrm{Lu} 4-\mathrm{A} 12$ \\
\hline $137.63(18)$ & $\mathrm{O} 8^{\mathrm{v}}-\mathrm{Lu} 4-\mathrm{A} 12$ \\
\hline 95.47 (17) & $\mathrm{O} 1^{\mathrm{ii}}-\mathrm{Lu} 4-\mathrm{A} 12$ \\
\hline $149.10(13)$ & 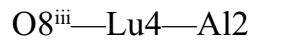 \\
\hline 37.17 (13) & $\mathrm{O} 4^{\mathrm{ix}}-\mathrm{Lu} 4-\mathrm{Lu} 3^{\mathrm{iii}}$ \\
\hline $125.28(5)$ & $\mathrm{O} 9-\mathrm{Lu} 4-\mathrm{Lu} 3^{\mathrm{iii}}$ \\
\hline $65.26(5)$ & $\mathrm{O}^{\mathrm{ii}}-\mathrm{Lu} 4-\mathrm{Lu}^{3 \mathrm{iii}}$ \\
\hline $139.2(2)$ & $\mathrm{O} 8^{\mathrm{v}}-\mathrm{Lu} 4-\mathrm{Lu}^{\mathrm{iii}}$ \\
\hline $37.22(14)$ & 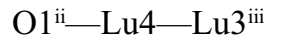 \\
\hline 85.85 (17) & 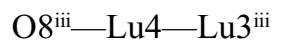 \\
\hline $135.01(15)$ & $\mathrm{A} 12-\mathrm{Lu} 4-\mathrm{Lu} 3^{\mathrm{iii}}$ \\
\hline 79.07 (14) & $\mathrm{O} 4^{\mathrm{ix}}-\mathrm{Lu} 4-\mathrm{Lu} 4^{\mathrm{x}}$ \\
\hline $91.80(13)$ & $\mathrm{O} 9-\mathrm{Lu} 4-\mathrm{Lu} 4^{\mathrm{x}}$ \\
\hline 39.37 (13) & 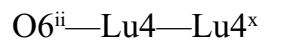 \\
\hline $86.95(5)$ & $\mathrm{O} 8^{\mathrm{v}}-\mathrm{Lu} 4-\mathrm{Lu} 4^{\mathrm{x}}$ \\
\hline $57.05(4)$ & 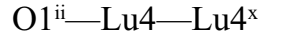 \\
\hline $59.875(14)$ & $\mathrm{O} 8^{\mathrm{iii}-\mathrm{Lu} 4-\mathrm{Lu}^{\mathrm{x}}}$ \\
\hline $36.30(13)$ & $\mathrm{A} 12-\mathrm{Lu} 4-\mathrm{Lu} 4^{\mathrm{x}}$ \\
\hline $143.97(15)$ & 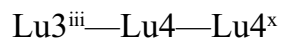 \\
\hline $110.32(15)$ & $\mathrm{O} 4^{\mathrm{ix}}-\mathrm{Lu} 4-\mathrm{Lu} 2^{\mathrm{ix}}$ \\
\hline
\end{tabular}

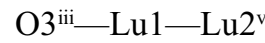

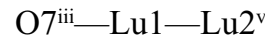

$$
\begin{aligned}
& \mathrm{O} 5^{\mathrm{iv}}-\mathrm{Lu} 1-\mathrm{Lu} 2^{\mathrm{v}} \\
& \mathrm{O} 2-\mathrm{Lu} 1-\mathrm{Lu} 2^{\mathrm{v}} \\
& \mathrm{O} 1-\mathrm{Lu} 1-\mathrm{Lu} 2^{\mathrm{v}}
\end{aligned}
$$

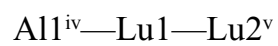

$$
\begin{aligned}
& \mathrm{A} 12^{\mathrm{iv}}-\mathrm{Lu} 1-\mathrm{Lu} 2^{\mathrm{v}}
\end{aligned}
$$

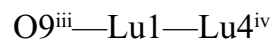

$$
\begin{aligned}
& \text { O6-Lu1-Lu4 }{ }^{\text {iv }} \\
& \text { O3 }{ }^{\text {iii }-\mathrm{Lu} 1-\mathrm{Lu} 4^{\mathrm{iv}}}
\end{aligned}
$$

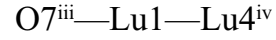

$$
\begin{aligned}
& \mathrm{O} 5^{\mathrm{iv}}-\mathrm{Lu} 1-\mathrm{Lu} 4^{\mathrm{iv}} \\
& \mathrm{O} 2-\mathrm{Lu} 1-\mathrm{Lu} 4^{\mathrm{iv}} \\
& \mathrm{O} 1-\mathrm{Lu} 1-\mathrm{Lu} 4^{\mathrm{iv}}
\end{aligned}
$$

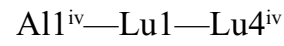

$$
\begin{aligned}
& \mathrm{A} 12^{\mathrm{iv}}-\mathrm{Lu} 1-\mathrm{Lu} 4^{\mathrm{iv}} \\
& \mathrm{Lu} 2^{\mathrm{v}}-\mathrm{Lu} 1-\mathrm{Lu} 4^{\mathrm{iv}} \\
& \text { O9iii-Lu1-Lu3 } \\
& \text { O6-Lu1-Lu3 } \\
& \mathrm{O} 3{ }^{i i i}-\mathrm{Lu} 1-\mathrm{Lu} 3
\end{aligned}
$$

$137.27(15)$

$95.71(15)$

$39.5(2)$

$41.12(12)$

$96.21(14)$

144.07 (12)

85.06 (13)

120.37 (4)

$87.25(5)$

$123.92(4)$

$64.036(14)$

$163.0(2)$

87.53 (18)

$77.1(2)$

84.7 (2)

$110.30(17)$

171.9 (2)

$75.4(2)$

$108.45(19)$

80.3 (2)

$100.0(2)$

$95.5(2)$

$79.9(2)$

$98.7(2)$

$79.7(3)$

170.9 (2)

$104.05(15)$

83.86 (13)

$91.72(14)$

92.45 (14)

30.48 (15)

$158.20(14)$

$135.90(15)$

39.61 (13)

92.25 (14)

91.67 (16)

$147.82(15)$

40.93 (18)

120.03 (4)

90.25 (15)

$96.21(15)$

137.47 (14)

40.44 (13)

$139.71(15)$

39.3 (2)

129.75 (4)

61.365 (15)

39.59 (15) 


$$
\begin{aligned}
& \text { O } 7^{i i i}-\mathrm{Lu} 1-\mathrm{Lu} 3 \\
& \mathrm{O} 5^{\mathrm{iv}}-\mathrm{Lu} 1-\mathrm{Lu} 3 \\
& \text { O2-Lu1-Lu3 } \\
& \text { O1-Lu1-Lu3 } \\
& \text { Al1 } 1 \text { - }-\mathrm{Lu} 1-\mathrm{Lu} 3 \\
& \mathrm{~A} 12^{\mathrm{iv}} \text { - Lu1-Lu3 } \\
& \text { Lu2 }{ }^{\mathrm{v}}-\mathrm{Lu} 1-\mathrm{Lu} 3 \\
& \mathrm{Lu} 4^{\mathrm{iv}} \text { - Lu1-Lu3 } \\
& \mathrm{O} 1^{\mathrm{vi}}-\mathrm{Lu} 2-\mathrm{O} 3^{\text {vii }} \\
& \mathrm{O} 1 \mathrm{vi}-\mathrm{Lu} 2-\mathrm{O} 2 \\
& \mathrm{O} 3^{\mathrm{vii}-\mathrm{Lu} 2-\mathrm{O} 2}
\end{aligned}
$$

\begin{tabular}{|c|c|}
\hline $106.94(16)$ & $\mathrm{O} 9-\mathrm{Lu} 4-\mathrm{Lu} 2^{\mathrm{ix}}$ \\
\hline $38.47(12)$ & $\mathrm{O} 6^{\mathrm{ii}}-\mathrm{Lu} 4-\mathrm{Lu} 2^{\mathrm{ix}}$ \\
\hline $83.91(13)$ & $\mathrm{O} 8^{\mathrm{v}}-\mathrm{Lu} 4-\mathrm{Lu} 2^{\mathrm{ix}}$ \\
\hline $81.20(13)$ & $\mathrm{O} 1^{\mathrm{ii}}-\mathrm{Lu} 4-\mathrm{Lu} 2^{\mathrm{ix}}$ \\
\hline $56.26(4)$ & $\mathrm{O} 8^{\mathrm{iii}}-\mathrm{Lu} 4-\mathrm{Lu} 2^{\mathrm{ix}}$ \\
\hline $55.19(4)$ & $\mathrm{A} 12-\mathrm{Lu} 4-\mathrm{Lu} 2^{\mathrm{ix}}$ \\
\hline $95.095(18)$ & 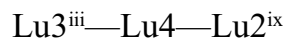 \\
\hline $112.083(15)$ & $\mathrm{Lu} 4^{\mathrm{x}}-\mathrm{Lu} 4-\mathrm{Lu} 2^{\mathrm{ix}}$ \\
\hline $81.6(2)$ & $\mathrm{O} 4^{\mathrm{ix}}-\mathrm{Lu} 4-\mathrm{Lu}^{\mathrm{v}}$ \\
\hline 89.32 (19) & O9-Lu4-Lu3 ${ }^{v}$ \\
\hline $169.1(2)$ & $\mathrm{O} 6^{\mathrm{ii}}-\mathrm{Lu} 4-\mathrm{Lu}^{\mathrm{v}}$ \\
\hline $164.5(2)$ & $\mathrm{O} 8^{v}-\mathrm{Lu} 4-\mathrm{Lu}^{\mathrm{v}}{ }^{\mathrm{v}}$ \\
\hline $113.97(17)$ & 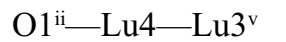 \\
\hline $75.2(2)$ & 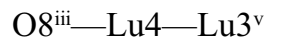 \\
\hline $91.6(2)$ & $\mathrm{A} 12-\mathrm{Lu} 4-\mathrm{Lu}^{\mathrm{v}}$ \\
\hline $88.2(2)$ & $\mathrm{Lu}^{3 \mathrm{ii}}-\mathrm{Lu} 4-\mathrm{Lu} 3^{\mathrm{v}}$ \\
\hline $98.0(2)$ & $\mathrm{Lu} 4^{\mathrm{x}}-\mathrm{Lu} 4-\mathrm{Lu} 3^{\mathrm{v}}$ \\
\hline $89.1(3)$ & $\mathrm{Lu} 2^{\mathrm{ix}}-\mathrm{Lu} 4-\mathrm{Lu} 3^{\mathrm{v}}$ \\
\hline $74.9(2)$ & $\mathrm{O} 4^{\mathrm{ix}}-\mathrm{Lu} 4-\mathrm{Lu} 1^{\mathrm{ii}}$ \\
\hline $92.4(2)$ & O9-Lu4-Lu $1^{\mathrm{ii}}$ \\
\hline $79.5(2)$ & $\mathrm{O} 6^{\mathrm{ii}}-\mathrm{Lu} 4-\mathrm{Lu} 1^{\mathrm{ii}}$ \\
\hline $103.2(2)$ & $\mathrm{O} 8^{\mathrm{v}}-\mathrm{Lu} 4-\mathrm{Lu} 1^{\mathrm{ii}}$ \\
\hline $166.2(2)$ & $\mathrm{O} 1^{\mathrm{ii}}-\mathrm{Lu} 4-\mathrm{Lu} 1^{\mathrm{ii}}$ \\
\hline 44.59 (16) & $\mathrm{O}{ }^{\mathrm{iii}}-\mathrm{Lu} 4-\mathrm{Lu} 1^{\mathrm{ii}}$ \\
\hline $37.11(17)$ & $\mathrm{A} 12-\mathrm{Lu} 4-\mathrm{Lu} 1^{\mathrm{ii}}$ \\
\hline $133.86(14)$ & $\mathrm{Lu} 3^{\mathrm{iii}}-\mathrm{Lu} 4-\mathrm{Lu} 1^{\mathrm{ii}}$ \\
\hline $150.92(17)$ & $\mathrm{Lu} 4^{\mathrm{x}}-\mathrm{Lu} 4-\mathrm{Lu} 1^{\mathrm{ii}}$ \\
\hline $87.2(2)$ & $\mathrm{Lu} 2^{\mathrm{ix}}-\mathrm{Lu} 4-\mathrm{Lu} 1^{\mathrm{ii}}$ \\
\hline 84.98 (14) & $\mathrm{Lu}^{\mathrm{v}}-\mathrm{Lu} 4-\mathrm{Lu} 1^{\mathrm{ii}}$ \\
\hline $38.06(15)$ & $\mathrm{A} 12^{\mathrm{iv}}-\mathrm{O} 1-\mathrm{Lu} 2^{\mathrm{v}}$ \\
\hline $86.66(16)$ & $\mathrm{A} 12^{\mathrm{iv}}-\mathrm{O} 1-\mathrm{Lu} 4^{\mathrm{iv}}$ \\
\hline $82.52(14)$ & $\mathrm{Lu} 2^{\mathrm{v}}-\mathrm{O} 1-\mathrm{Lu} 4^{\mathrm{iv}}$ \\
\hline $137.89(15)$ & $\mathrm{A} 12^{\mathrm{iv}}-\mathrm{O} 1-\mathrm{Lu} 1$ \\
\hline $129.58(17)$ & $\mathrm{Lu} 2^{\mathrm{v}}-\mathrm{O} 1-\mathrm{Lu} 1$ \\
\hline $36.84(14)$ & $\mathrm{Lu} 4^{\mathrm{iv}}-\mathrm{O} 1-\mathrm{Lu} 1$ \\
\hline $60.420(13)$ & $\mathrm{A} 11^{\mathrm{iv}}-\mathrm{O} 2-\mathrm{Lu} 2$ \\
\hline 85.18 (17) & $\mathrm{A} 11^{\mathrm{iv}}-\mathrm{O} 2-\mathrm{Lu}^{\mathrm{iv}}$ \\
\hline $136.93(14)$ & $\mathrm{Lu} 2-\mathrm{O} 2-\mathrm{Lu}^{\mathrm{iv}}$ \\
\hline $35.36(14)$ & $\mathrm{A} 11^{\mathrm{iv}}-\mathrm{O} 2-\mathrm{Lu} 1$ \\
\hline $83.02(17)$ & $\mathrm{Lu} 2-\mathrm{O} 2-\mathrm{Lu} 1$ \\
\hline $133.14(18)$ & $\mathrm{Lu} 3^{\mathrm{iv}}-\mathrm{O} 2-\mathrm{Lu} 1$ \\
\hline $44.56(15)$ & Al1-O3-Lu2 $2^{\mathrm{vii}}$ \\
\hline $119.653(15)$ & $\mathrm{A} 11-\mathrm{O} 3-\mathrm{Lu} 1^{\mathrm{iii}}$ \\
\hline $59.233(14)$ & $\mathrm{Lu} 2^{\mathrm{vii}}-\mathrm{O} 3-\mathrm{Lu} 1^{\mathrm{iii}}$ \\
\hline $128.85(16)$ & $\mathrm{A} 11^{\mathrm{xi}}-\mathrm{O} 4-\mathrm{Lu} 4^{\mathrm{viii}}$ \\
\hline $149.48(17)$ & $\mathrm{A} 11^{\mathrm{xi}}-\mathrm{O} 4-\mathrm{Lu} 2$ \\
\hline $40.30(14)$ & $\mathrm{Lu} 44^{\mathrm{vii}}-\mathrm{O} 4-\mathrm{Lu} 2$ \\
\hline $35.63(17)$ & $\mathrm{Al} 11^{\mathrm{xi}}-\mathrm{O} 4-\mathrm{Lu} 3^{\mathrm{iv}}$ \\
\hline
\end{tabular}

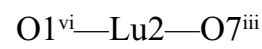

$$
\begin{aligned}
& \mathrm{O} 3^{\text {vii }-\mathrm{Lu} 2-\mathrm{O} 7^{\mathrm{iii}}} \\
& \mathrm{O} 2-\mathrm{Lu} 2-\mathrm{O} 7^{\mathrm{iii}} \\
& \mathrm{O} 1^{\mathrm{vi}}-\mathrm{Lu} 2-\mathrm{O} 8^{\mathrm{vii}} \\
& \mathrm{O}^{\mathrm{vii}}-\mathrm{Lu} 2-\mathrm{O}^{\mathrm{vii}} \\
& \mathrm{O} 2-\mathrm{Lu} 2-\mathrm{O} 8^{\mathrm{vii}} \\
& \mathrm{O} 7^{\mathrm{iii}}-\mathrm{Lu} 2-\mathrm{O}^{\mathrm{vii}} \\
& \mathrm{O}^{\mathrm{vi}}-\mathrm{Lu} 2-\mathrm{O} 4 \\
& \mathrm{O} 3{ }^{\mathrm{vi}-\mathrm{Lu} 2-\mathrm{O} 4} \\
& \mathrm{O} 2-\mathrm{Lu} 2-\mathrm{O} 4
\end{aligned}
$$

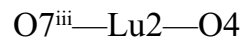

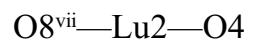

$$
\begin{aligned}
& \mathrm{O} 1^{\mathrm{vi}}-\mathrm{Lu} 2-\mathrm{Lu} 1^{\mathrm{vi}} \\
& \mathrm{O} 3^{\text {vii_Lu2-Lu1 }}{ }^{\text {vi }} \\
& \mathrm{O} 2-\mathrm{Lu} 2-\mathrm{Lu} 1^{\mathrm{vi}}
\end{aligned}
$$

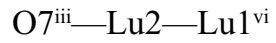

$$
\begin{aligned}
& \mathrm{O} 8^{\mathrm{vii}}-\mathrm{Lu} 2-\mathrm{Lu} 1^{\mathrm{vi}} \\
& \mathrm{O} 4-\mathrm{Lu} 2-\mathrm{Lu} 1^{\mathrm{vi}} \\
& \mathrm{O} 1^{\text {vi }-\mathrm{Lu} 2-\mathrm{Lu} 4^{\mathrm{viii}}} \\
& \mathrm{O} 3^{\text {vii }-\mathrm{Lu} 2-\mathrm{Lu} 4^{\mathrm{viii}}} \\
& \mathrm{O} 2-\mathrm{Lu} 2-\mathrm{Lu} 4^{\mathrm{viii}} \\
& \mathrm{O} 7^{\mathrm{iii}}-\mathrm{Lu} 2-\mathrm{Lu} 4^{\mathrm{viii}} \\
& \mathrm{O} 8^{\mathrm{vii}}-\mathrm{Lu} 2-\mathrm{Lu} 4^{\mathrm{viii}} \\
& \text { O4-Lu2-Lu4 } 4^{\text {viii }} \\
& \mathrm{Lu} 1^{\mathrm{vi}}-\mathrm{Lu} 2-\mathrm{Lu} 4^{\mathrm{viii}} \\
& \mathrm{O} 1^{\mathrm{vi}}-\mathrm{Lu} 2-\mathrm{Lu}^{\mathrm{iv}} \\
& \mathrm{O}^{\mathrm{vii}}-\mathrm{Lu} 2-\mathrm{Lu}^{\mathrm{iv}} \\
& \mathrm{O} 2-\mathrm{Lu} 2-\mathrm{Lu}^{\mathrm{iv}}
\end{aligned}
$$

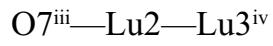

$$
\begin{aligned}
& \mathrm{O} 8^{\text {vii }-\mathrm{Lu} 2-\mathrm{Lu}^{\mathrm{iv}}} \\
& \mathrm{O} 4-\mathrm{Lu} 2-\mathrm{Lu} 3^{\mathrm{iv}} \\
& \mathrm{Lu} 1^{\mathrm{vi}}-\mathrm{Lu} 2-\mathrm{Lu}^{\mathrm{iv}} \\
& \mathrm{Lu} 4^{\mathrm{viii}}-\mathrm{Lu} 2-\mathrm{Lu} 3^{\text {iv }} \\
& \mathrm{O} 1^{\mathrm{vi}}-\mathrm{Lu} 2-\mathrm{Lu} 1 \\
& \text { O3 }{ }^{\text {vii-Lu2-Lu1 }} \\
& \mathrm{O} 2-\mathrm{Lu} 2-\mathrm{Lu} 1
\end{aligned}
$$

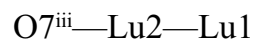

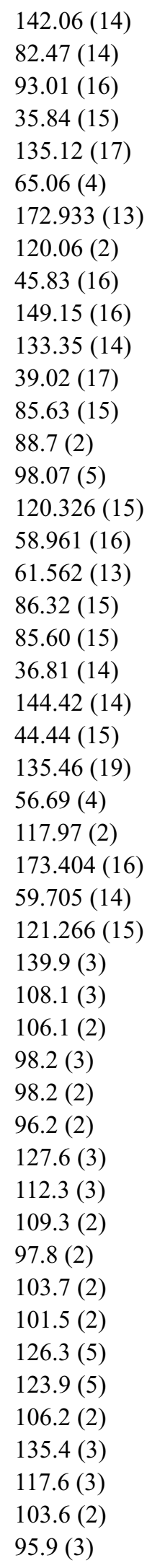




\begin{tabular}{|c|c|c|c|}
\hline 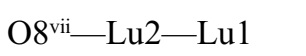 & $88.6(2)$ & $\mathrm{Lu} 4^{\mathrm{viii}}-\mathrm{O} 4-\mathrm{Lu}^{\mathrm{iv}}$ & $96.4(2)$ \\
\hline $\mathrm{O} 4-\mathrm{Lu} 2-\mathrm{Lu} 1$ & $97.74(14)$ & $\mathrm{Lu} 2-\mathrm{O} 4-\mathrm{Lu} 3^{\mathrm{iv}}$ & $95.9(2)$ \\
\hline Lu1 ${ }^{\mathrm{vi}-\mathrm{Lu} 2-\mathrm{Lu} 1}$ & $172.025(12)$ & $\mathrm{A} 11-\mathrm{O} 5-\mathrm{A} 12$ & $134.9(3)$ \\
\hline 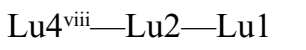 & $118.072(14)$ & $\mathrm{A} 11-\mathrm{O} 5-\mathrm{Lu} 3^{\mathrm{ii}}$ & $105.3(3)$ \\
\hline $\mathrm{Lu}^{3}{ }^{\mathrm{iv}}-\mathrm{Lu} 2-\mathrm{Lu} 1$ & $59.438(13)$ & $\mathrm{A} 12-\mathrm{O} 5-\mathrm{Lu} 3^{\mathrm{ii}}$ & $103.1(3)$ \\
\hline $\mathrm{O} 1^{\mathrm{vi}}-\mathrm{Lu} 2-\mathrm{Lu} 4^{\mathrm{iii}}$ & $124.70(15)$ & $\mathrm{A} 11-\mathrm{O} 5-\mathrm{Lu} 1^{\mathrm{ii}}$ & $102.6(3)$ \\
\hline 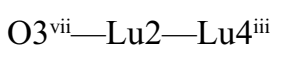 & $102.42(15)$ & $\mathrm{A} 12-\mathrm{O} 5-\mathrm{Lu} 1^{\mathrm{ii}}$ & $104.6(3)$ \\
\hline $\mathrm{O} 2-\mathrm{Lu} 2-\mathrm{Lu} 4^{\mathrm{iii}}$ & $87.60(14)$ & $\mathrm{Lu} 3^{\mathrm{ii}}-\mathrm{O} 5-\mathrm{Lu} 1^{\mathrm{ii}}$ & $102.39(18)$ \\
\hline $\mathrm{O} 7^{\mathrm{iii}}-\mathrm{Lu} 2-\mathrm{Lu} 4^{\mathrm{iii}}$ & $54.38(15)$ & $\mathrm{A} 12^{\mathrm{xi}}-\mathrm{O} 6-\mathrm{Lu} 1$ & 122.0 \\
\hline 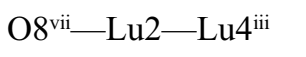 & $34.8(2)$ & $\mathrm{A} 12^{\mathrm{xi}}-\mathrm{O} 6-\mathrm{Lu} 4^{\mathrm{iv}}$ & $125.6(3)$ \\
\hline $\mathrm{O} 4-\mathrm{Lu} 2-\mathrm{Lu} 4^{\mathrm{iii}}$ & $156.67(14)$ & $\mathrm{Lu} 1-\mathrm{O} 6-\mathrm{Lu} 4^{\mathrm{iv}}$ & $106.0(2)$ \\
\hline 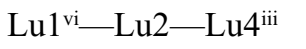 & $117.607(14)$ & $\mathrm{A} 12^{\mathrm{xi}}-\mathrm{O} 6-\mathrm{Lu}^{\mathrm{iv}}$ & $95.4(2)$ \\
\hline $\mathrm{Lu} 4^{\mathrm{viii}}-\mathrm{Lu} 2-\mathrm{Lu} 4^{\mathrm{iii}}$ & $159.792(10)$ & $\mathrm{Lu} 1-\mathrm{O} 6-\mathrm{Lu}^{\mathrm{iv}}$ & $99.6(2)$ \\
\hline $\mathrm{Lu} 3^{\mathrm{iv}}-\mathrm{Lu} 2-\mathrm{Lu} 4^{\mathrm{iii}}$ & $118.642(17)$ & $\mathrm{Lu} 4^{\mathrm{iv}}-\mathrm{O} 6-\mathrm{Lu} 3^{\mathrm{iv}}$ & $100.7(2)$ \\
\hline $\mathrm{Lu} 1-\mathrm{Lu} 2-\mathrm{Lu} 4^{\mathrm{iii}}$ & $60.716(12)$ & $\mathrm{A} 12-\mathrm{O} 7-\mathrm{Lu} 2^{\mathrm{iii}}$ & $126.1(5)$ \\
\hline $\mathrm{O} 1^{\mathrm{vi}}-\mathrm{Lu} 2-\mathrm{Lu} 3^{\mathrm{vii}}$ & $85.85(16)$ & $\mathrm{A} 12-\mathrm{O} 7-\mathrm{Lu} 1^{\mathrm{iii}}$ & $123.6(5)$ \\
\hline $\mathrm{O}{ }^{\mathrm{vii}}-\mathrm{Lu} 2-\mathrm{Lu}^{\mathrm{vii}}$ & $56.75(14)$ & $\mathrm{Lu} 2^{\mathrm{iii}}-\mathrm{O} 7-\mathrm{Lu} 1^{\mathrm{iii}}$ & $109.0(2)$ \\
\hline $\mathrm{O} 2-\mathrm{Lu} 2-\mathrm{Lu}^{\mathrm{vii}}$ & $128.77(14)$ & $\mathrm{Lu} 4^{\mathrm{vi}}-\mathrm{O} 8-\mathrm{Lu} 2^{\mathrm{vii}}$ & $110.1(3)$ \\
\hline 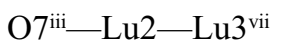 & $102.54(16)$ & $\mathrm{Lu} 44^{\mathrm{vi}}-\mathrm{O} 8-\mathrm{Lu} 3$ & $102.9(2)$ \\
\hline $\mathrm{O} 8^{\mathrm{vii}}-\mathrm{Lu} 2-\mathrm{Lu} 3^{\mathrm{vii}}$ & $31.5(2)$ & 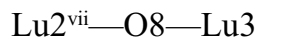 & $117.4(3)$ \\
\hline $\mathrm{O} 4-\mathrm{Lu} 2-\mathrm{Lu} 3^{\mathrm{vii}}$ & $146.03(14)$ & $\mathrm{Lu} 4^{\mathrm{vi}}-\mathrm{O} 8-\mathrm{Lu} 4^{\mathrm{iii}}$ & 100.3 \\
\hline $\mathrm{Lu} 1^{\mathrm{vi}}-\mathrm{Lu} 2-\mathrm{Lu}^{\mathrm{vii}}$ & $62.045(13)$ & $\mathrm{Lu} 2^{\mathrm{vii}}-\mathrm{O} 8-\mathrm{Lu} 4^{\mathrm{iii}}$ & $125.0(2)$ \\
\hline $\mathrm{Lu} 4^{\mathrm{viii}}-\mathrm{Lu} 2-\mathrm{Lu} 3^{\mathrm{vii}}$ & $119.182(17)$ & $\mathrm{Lu} 3-\mathrm{O} 8-\mathrm{Lu} 4^{\mathrm{iii}}$ & $97.9(2)$ \\
\hline $\mathrm{Lu}^{\mathrm{iv}}-\mathrm{Lu} 2-\mathrm{Lu} 3^{\mathrm{vii}}$ & $161.782(11)$ & $\mathrm{Lu} 1^{\mathrm{iii}}-\mathrm{O} 9-\mathrm{Lu} 3$ & $120.0(4)$ \\
\hline Lu1-Lu2-Lu3 ${ }^{\mathrm{vii}}$ & $116.038(15)$ & $\mathrm{Lu} 1{ }^{\mathrm{iii}}-\mathrm{O} 9-\mathrm{Lu} 4$ & $113.8(3)$ \\
\hline 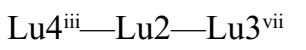 & $55.607(13)$ & $\mathrm{Lu} 3-\mathrm{O} 9-\mathrm{Lu} 4$ & $110.2(2)$ \\
\hline $\mathrm{O} 2^{\mathrm{ii}-\mathrm{Lu} 3-\mathrm{O} 9}$ & $102.6(2)$ & $\mathrm{Lu} 1^{\mathrm{iii}}-\mathrm{O} 9-\mathrm{Lu} 3^{\mathrm{iii}}$ & $108.2(2)$ \\
\hline 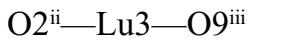 & $176.7(3)$ & Lu3-O9-Lu3 $3^{\mathrm{iii}}$ & $101.0(3)$ \\
\hline $\mathrm{O} 9-\mathrm{Lu} 3-\mathrm{O} 9^{\mathrm{iii}}$ & $79.0(3)$ & $\mathrm{Lu} 4-\mathrm{O} 9-\mathrm{Lu}^{\mathrm{iii}}$ & $100.9(3)$ \\
\hline
\end{tabular}

Symmetry codes: (i) $x, y, z-1$; (ii) $x,-y+1 / 2, z-1 / 2$; (iii) $-x+1,-y,-z+1$; (iv) $x,-y+1 / 2, z+1 / 2$; (v) $x+1, y, z$; (vi) $x-1, y, z$; (vii) $-x,-y,-z+1$; (viii) $x-1$, $-y+1 / 2, z+1 / 2$; (ix) $x+1,-y+1 / 2, z-1 / 2$; (x) $-x+2,-y,-z+1$; (xi) $x, y, z+1$. 